\title{
Prenylated Flavonoids from the Roots of Tephrosia rhodesica
}

\author{
Yoseph Atilaw, Lois Muiva-Mutisya, Jonathan Bogaerts, Sandra Duffy, Arto Valkonen, \\ Matthias Heydenreich, Vicky M. Avery, Kari Rissanen, Máté Erdélyi,* and Abiy Yenesew*
}

Cite This: https://dx.doi.org/10.1021/acs.jnatprod.0c00245

ABSTRACT: Five new compounds-rhodimer (1), rhodiflavan A (2), rhodiflavan B (3), rhodiflavan C (4), and rhodacarpin (5) - along with 16 known secondary metabolites, were isolated from the $\mathrm{CH}_{2} \mathrm{Cl}_{2}-\mathrm{CH}_{3} \mathrm{OH}(1: 1)$ extract of the roots of Tephrosia rhodesica. They were identified by NMR spectroscopic, mass spectrometric, X-ray crystallographic, and ECD spectroscopic analyses. The crude extract and the isolated compounds 2-5, 9, 15, and 21 showed activity $\left(100 \%\right.$ at $10 \mu \mathrm{g}$ and $\left.\mathrm{IC}_{50}=5-15 \mu \mathrm{M}\right)$ against the chloroquine-sensitive (3D7) strain of Plasmodium falciparum.
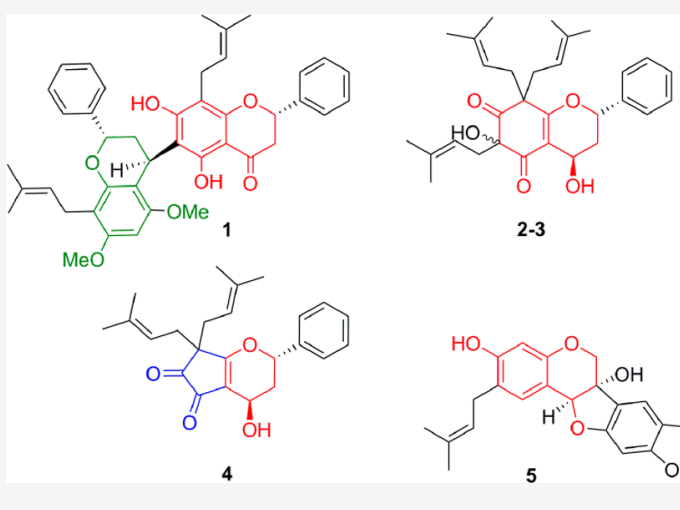

Tephrosia (Leguminosae) is a tropical and subtropical genus consisting of more than 400 species, 30 of which are native to Kenya. ${ }^{1}$ These have provided a variety of new flavonoids, including flavanones, flavones, chalcones, pterocarpans, and rotenoids, most of them prenylated and showing promising antiplasmodial, antifeedant, antileishmanial, estrogenic, antitumor, and antimicrobial activities. ${ }^{2-6}$ As part of our ongoing work on the genus Tephrosia, ${ }^{6,7}$ we now report the first phytochemical investigation of the roots of Tephrosia rhodesica, which showed antiplasmodial activity. The investigation of the root extract led to the isolation of five new compounds $(\mathbf{1}-\mathbf{5})$ and 16 known compounds (6-21). Herein, we describe their isolation, characterization, and activity against the chloroquinesensitive (3D7) strain of Plasmodium falciparum.

\section{RESULTS AND DISCUSSION}

Applying silica gel gravity column chromatography, followed by Sephadex LH-20 gel filtration and preparative reverse-phase HPLC, 21 secondary metabolites were isolated from the roots of $T$. rhodesica. The structures of the isolated secondary metabolites were determined by NMR spectroscopic, mass spectrometric, and single-crystal X-ray diffractometric analyses. In addition to the five new secondary metabolites $(\mathbf{1 - 5})$, the known tephrowatsin B (6), ${ }^{8}$ tephrinone $(7),{ }^{9}$ glabranin $(8),{ }^{10}$ quercetol B (9), ${ }^{11}$ maackiain (10), ${ }^{12}$ 6a-hydroxymaackiain (11) ${ }^{13}$ pisatin (12), ${ }^{14}$ tephrosin $(\mathbf{1 3}),{ }^{15}$ rotenone $(\mathbf{1 4}),{ }^{15} 6$ hydroxyrotenone $(\mathbf{1 5}),{ }^{16}$ 12a-hydroxyrotenone (16), ${ }^{17}$ hildecarpin (17), ${ }^{18}$ 3-hydroxy-2-methoxy-8,9-methylenedioxypterocarpene (18), ${ }^{19}$ isoliquirtigenin (19), ${ }^{20} \mathrm{D}$-pinitol (20), ${ }^{21}$ and tephrowatsin A (21) ${ }^{8}$ were identified by comparison of their observed and reported spectroscopic data (see pages S22-S34 in the Supporting Information).
Compound 1 was isolated as white crystals from $\mathrm{CH}_{2} \mathrm{Cl}_{2}-$ $\mathrm{CH}_{3} \mathrm{OH}$ (1:1) solution. Its molecular formula was deduced as $\mathrm{C}_{42} \mathrm{H}_{44} \mathrm{O}_{7}$ based on HREIMS ([M $]^{+} \mathrm{m} / z$ 660.3095, calcd 660.3087) and NMR data analyses (see Table 1 , as well as Figures $\mathrm{S} 1-\mathrm{S} 6$ in the Supporting Information). The ${ }^{1} \mathrm{H}$ NMR signals at $\delta_{\mathrm{H}} 5.43(\mathrm{H}-2), 5.06\left(\mathrm{H}-2^{\prime \prime \prime}\right), 2.84$ and $3.07(\mathrm{H}-3 \mathrm{a}-\mathrm{b})$, 2.18 and $2.31\left(\mathrm{H}-3^{\prime \prime \prime}\right)$, and $4.66\left(\mathrm{H}-4^{\prime \prime \prime}\right)$, along with the ${ }^{13} \mathrm{C}$ NMR signals at $\delta_{\mathrm{C}} 78.6(\mathrm{C}-2), 75.4\left(\mathrm{C}-2^{\prime \prime \prime}\right), 43.7(\mathrm{C}-3), 36.9$ (C-3"' $), 196.3(\mathrm{C}-4)$, and 26.8 (C-4 $\left.{ }^{\prime \prime \prime}\right)$ were consistent with a flavanone-flavan dimer core structure ${ }^{22,23}$ that was corroborated by the UV absorptions of $\lambda_{\max }=230,290$, and $350 \mathrm{~nm}$. The NMR data (Table 1) indicated the presence of two sets of unsubstituted aromatic rings, two sets of prenyl groups, two hydroxy, and two methoxy groups. This is consistent with a 5hydroxyflavanone moiety possessing an unsubstituted ring $\mathrm{B}$, along with a ring $\mathrm{A}$ substituted with prenyl at C-8 $\left(\delta_{\mathrm{C}} 108.1\right)$ and two hydroxy groups, $\delta_{\mathrm{H}} 12.61(\mathrm{HO}-5)$ and $6.80(\mathrm{HO}-7)$ at C-5 $\left(\delta_{\mathrm{C}} 159.3\right)$ and C-7 $\left(\delta_{\mathrm{C}} 162.8\right)$. Accordingly, C-6 $\left(\delta_{\mathrm{C}}\right.$ $109.8)$ connects the flavanone to the flavan moiety. Whereas the placement of a hydroxy group at C-7 was based on biogenetic considerations, ${ }^{24}$ and on the chemical shift of the HO-5 hydrogen $\left(\delta_{\mathrm{H}} 12.61\right)$, suggesting its involvement in an intramolecular hydrogen bond; the placement of the prenyl group at C-8 $\left(\delta_{\mathrm{C}} 108.1\right)$ rather than at C-6 $\left(\delta_{\mathrm{C}} 109.8\right)$ was

Received: March 5, 2020 


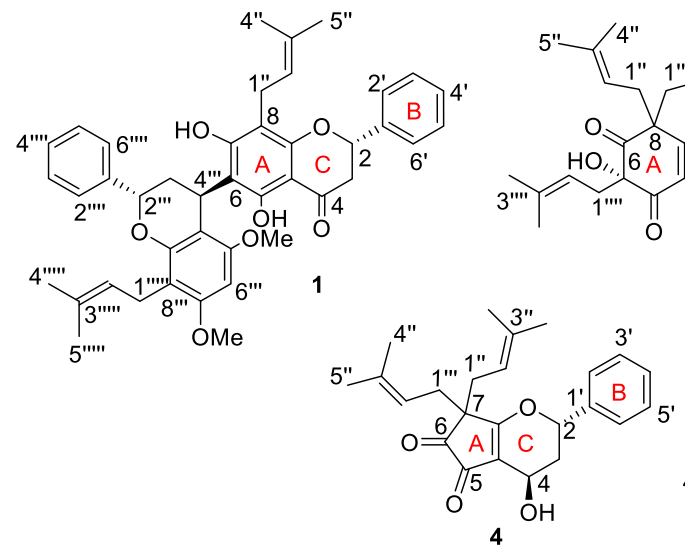

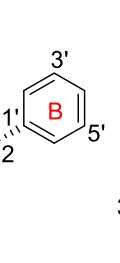

2

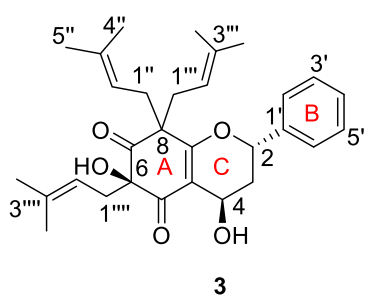

3

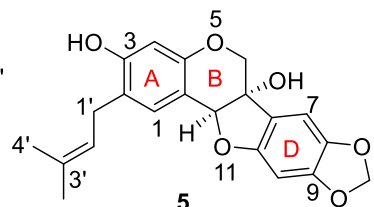

Table 1. NMR Spectroscopic Data (800 $\left.\mathrm{MHz}, \mathrm{CDCl}_{3}\right)$ for Rhodimer (1)

\begin{tabular}{|c|c|c|c|}
\hline position & $\delta_{\mathrm{C}, \text { type }}$ & $\delta_{\mathrm{H}}(J$, in $\mathrm{Hz})$ & HMBC \\
\hline 2 & 78.6, $\mathrm{CH}$ & 5.43 , dd $(2.9,13.1)$ & $\mathrm{C}-4, \mathrm{C}-1^{\prime}, \mathrm{C}-2^{\prime} / 6^{\prime}, \mathrm{C}-8 \mathrm{a}$ \\
\hline 3 & 43.7, $\mathrm{CH}_{2}$ & 2.84, dd $(2.9,17.1) 3.07$, dd $(13.1,17.1)$ & $\mathrm{C}-2, \mathrm{C}-4, \mathrm{C}-1^{\prime}, \mathrm{C}-4, \mathrm{C}-4 \mathrm{a}, \mathrm{C}-1^{\prime}$ \\
\hline 4 & 196.3, $\mathrm{C}=\mathrm{O}$ & & \\
\hline $4 a$ & 100.4, C & & \\
\hline 5 & $159.3, \mathrm{C}-\mathrm{O}$ & & \\
\hline 6 & $109.8, \mathrm{C}$ & & \\
\hline 7 & $162.8, \mathrm{C}-\mathrm{O}$ & & \\
\hline 8 & 108.1, C & & \\
\hline $8 \mathrm{a}$ & 157.6, C-O & & \\
\hline $1^{\prime}$ & 139.1, C & & \\
\hline $2^{\prime} / 6^{\prime}$ & $125.9, \mathrm{CH}$ & $7.47, \mathrm{~m}$ & $\mathrm{C}-2, \mathrm{C}-2^{\prime} / 6^{\prime}, \mathrm{C}-3^{\prime} / 5^{\prime}$ \\
\hline $3^{\prime} / 5^{\prime}$ & 128.7, $\mathrm{CH}$ & $7.43, \mathrm{~m}$ & $\mathrm{C}-1^{\prime}, \mathrm{C}-3^{\prime} / 5^{\prime}$ \\
\hline $4^{\prime}$ & $128.5, \mathrm{CH}$ & $7.39, \mathrm{~m}$ & $\mathrm{C}-2^{\prime} / 6^{\prime}$ \\
\hline $1^{\prime \prime}$ & $21.8, \mathrm{CH}_{2}$ & 3.18 , br t & $\mathrm{C}-7, \mathrm{C}-8, \mathrm{C}-8 \mathrm{a}, \mathrm{C}-3^{\prime \prime}, \mathrm{C}-2^{\prime \prime}$ \\
\hline $2^{\prime \prime}$ & $122.5, \mathrm{CH}$ & 5.15 , br t & $\mathrm{C}-1^{\prime \prime}, \mathrm{C}-4^{\prime \prime}, \mathrm{C}-5^{\prime \prime}$ \\
\hline $3^{\prime \prime}$ & 131.6, C & & \\
\hline $4^{\prime \prime}$ & $17.8, \mathrm{CH}_{3}$ & $1.57, \mathrm{~s}$ & $\mathrm{C}-3^{\prime \prime}, \mathrm{C}-2^{\prime \prime}, \mathrm{C}-5^{\prime}$ \\
\hline $5^{\prime \prime}$ & $25.8, \mathrm{CH}_{3}$ & $1.65, \mathrm{~s}$ & $\mathrm{C}-3^{\prime \prime}, \mathrm{C}-2^{\prime \prime}, \mathrm{C}-4^{\prime}$ \\
\hline $\mathrm{OH}-5$ & & $12.61, \mathrm{~s}$ & C-5, C-6, C-4a, C-4, C-7 \\
\hline $\mathrm{OH}-7$ & & $6.80, \mathrm{~s}$ & C-6, C-8, C-8a, C-7, C-4a \\
\hline $2^{\prime \prime \prime}$ & 75.4, $\mathrm{CH}$ & 5.06 , dd $(2.0,11.5)$ & $\mathrm{C}-4^{\prime \prime \prime}, \mathrm{C}-1^{\prime \prime \prime \prime}, \mathrm{C}-2^{\prime \prime \prime \prime} / 6^{\prime \prime \prime \prime}, \mathrm{C}-8 \mathrm{a}$ \\
\hline $3^{\prime \prime \prime}$ & $36.9, \mathrm{CH}_{2}$ & $2.18, \mathrm{dt}(2.0,6.0,14.0) 2.31, \mathrm{dt}(2.0,11.5,14.0)$ & $\mathrm{C}-1^{\prime \prime \prime \prime}, \mathrm{C}-4^{\prime \prime \prime}, \mathrm{C}-6, \mathrm{C}-4^{\prime \prime \prime}, \mathrm{C}-4^{\prime \prime \prime}{ }^{\prime \prime}, \mathrm{C}-6$ \\
\hline $4^{\prime \prime \prime}$ & 26.8, $\mathrm{CH}$ & 4.66, dd $(2.0,6.0)$ & $\mathrm{C}-2^{\prime \prime \prime}, \mathrm{C}-3^{\prime \prime \prime}, \mathrm{C}-4^{\prime \prime \prime}$ a, C- $5^{\prime \prime \prime}, \mathrm{C}-8^{\prime \prime \prime}$ a, C-5, C-6, C-7 \\
\hline $4^{\prime \prime \prime} \mathrm{a}$ & $102.8, \mathrm{C}$ & & \\
\hline $5^{\prime \prime \prime}$ & $157.5, \mathrm{C}-\mathrm{O}$ & & \\
\hline $6^{\prime \prime \prime}$ & $88.4, \mathrm{CH}$ & $6.17, \mathrm{~s}$ & $\mathrm{C}-4^{\prime \prime \prime}, \mathrm{C}-4^{\prime \prime \prime}$ a $, \mathrm{C}-5^{\prime \prime \prime}, \mathrm{C}-7^{\prime \prime \prime}, \mathrm{C}-8^{\prime \prime \prime}, \mathrm{C}-1^{\prime \prime \prime \prime \prime}(\mathrm{w})$ \\
\hline $7^{\prime \prime \prime}$ & $158.6, \mathrm{C}-\mathrm{O}$ & & \\
\hline $8^{\prime \prime \prime}$ & $110.5, \mathrm{C}$ & & \\
\hline $8^{\prime \prime \prime} \mathrm{a}$ & $154.3, \mathrm{C}-\mathrm{O}$ & & \\
\hline $1^{\prime \prime \prime \prime}$ & 141.4, C & & \\
\hline $2^{\prime \prime \prime \prime} / 6^{\prime \prime \prime \prime}$ & 126.0, $\mathrm{CH}$ & $7.38, \mathrm{~m}$ & $\mathrm{C}-2^{\prime \prime \prime}, \mathrm{C}-2^{\prime \prime \prime \prime} / 6^{\prime \prime \prime \prime}, \mathrm{C}-3^{\prime \prime \prime \prime} / 5^{\prime \prime \prime \prime}$ \\
\hline $3^{\prime \prime \prime \prime} / 5^{\prime \prime \prime \prime}$ & $128.3, \mathrm{CH}$ & $7.34, \mathrm{~m}$ & $\mathrm{C}-3^{\prime \prime \prime \prime} / 5^{\prime \prime \prime \prime}, \mathrm{C}-1^{\prime \prime \prime \prime}$ \\
\hline $4^{\prime \prime \prime \prime}$ & 127.6, $\mathrm{CH}$ & $7.29, \mathrm{~m}$ & $\mathrm{C}-2^{\prime \prime \prime \prime} / 6^{\prime \prime \prime \prime}$ \\
\hline $1^{\prime \prime \prime \prime \prime \prime}$ & 21.9, $\mathrm{CH}_{2}$ & 3.35, br t & $\mathrm{C}-8^{\prime \prime \prime}, \mathrm{C}-8 \mathrm{a}^{\prime \prime \prime}, \mathrm{C}-7^{\prime \prime \prime}, \mathrm{C}-3^{\prime \prime \prime \prime \prime}, \mathrm{C}-2^{\prime \prime \prime \prime \prime}$ \\
\hline $2^{\prime \prime \prime \prime \prime}$ & 123.2, $\mathrm{CH}$ & 5.24 , br t & $\mathrm{C}-1^{\prime \prime \prime \prime \prime}, \mathrm{C}-4^{\prime \prime \prime \prime \prime}, \mathrm{C}-5^{\prime \prime \prime \prime \prime}$ \\
\hline $3^{\prime \prime \prime \prime \prime \prime}$ & $130.8, \mathrm{C}$ & & \\
\hline $4^{\prime \prime \prime \prime \prime \prime}$ & $17.8, \mathrm{CH}_{3}$ & $1.66, \mathrm{~s}$ & $\mathrm{C}-2^{\prime \prime \prime \prime \prime}, \mathrm{C}-3^{\prime \prime \prime \prime \prime}, \mathrm{C}-5^{\prime \prime \prime \prime \prime}$ \\
\hline $5^{\prime \prime \prime \prime \prime}$ & 25.9, $\mathrm{CH}_{3}$ & $1.68, \mathrm{~s}$ & $\mathrm{C}-2^{\prime \prime \prime \prime \prime}, \mathrm{C}-3^{\prime \prime \prime \prime \prime}, \mathrm{C}-4^{\prime \prime \prime \prime \prime}$ \\
\hline OMe-5"' & $55.9, \mathrm{C}-\mathrm{O}$ & $3.73, \mathrm{~s}$ & $\mathrm{C}-5^{\prime \prime \prime}$ \\
\hline OMe- $7^{\prime \prime \prime}$ & $55.8, \mathrm{C}-\mathrm{O}$ & $3.87, \mathrm{~s}$ & $\mathrm{C}-7^{\prime \prime \prime}$ \\
\hline
\end{tabular}

based on the HMBC correlation of $\mathrm{CH}_{2}-1^{\prime \prime}\left(\delta_{\mathrm{H}} 3.18\right)$ with C$7\left(\delta_{\mathrm{C}} 162.8\right), \mathrm{C}-8\left(\delta_{\mathrm{C}} 108.1\right), \mathrm{C}-8 \mathrm{a}\left(\delta_{\mathrm{C}} 157.6\right), \mathrm{C}-3^{\prime \prime}\left(\delta_{\mathrm{C}}\right.$ $131.6), \mathrm{C}-2^{\prime \prime}\left(\delta_{\mathrm{C}} 122.5\right)$, and of $\mathrm{H}-2\left(\delta_{\mathrm{H}} 5.43\right)$ with C-4 $\left(\delta_{\mathrm{C}}\right.$ $196.3), \mathrm{C}-1^{\prime}\left(\delta_{\mathrm{C}} 139.1\right), \mathrm{C}-2^{\prime} / \mathrm{C}-6^{\prime}\left(\delta_{\mathrm{C}} 125.9\right), \mathrm{C}-8 \mathrm{a}\left(\delta_{\mathrm{C}}\right.$
157.6). The identity of the other half of the molecule was established as a 5,7-dimethoxy-8-prenylflavan moiety based on the NMR data (Table 1). Hence, its ${ }^{1} \mathrm{H}$ NMR spectrum revealed the presence of an unsubstituted aromatic ring (ring 
E) $\left[\delta_{\mathrm{H}} 7.38\left(\mathrm{H}-2^{\prime \prime \prime \prime} / 6^{\prime \prime \prime \prime}\right), 7.34\left(\mathrm{H}-3^{\prime \prime \prime \prime} / 5^{\prime \prime \prime \prime}\right), 7.29(\mathrm{H}-\right.$ $\left.4^{\prime \prime \prime \prime}\right)$, two methoxy groups at $\delta_{\mathrm{H}} 3.73\left(\mathrm{OMe}-5^{\prime \prime \prime}\right)$ and 3.87 $\left(\mathrm{OMe}-7^{\prime \prime \prime}\right)$ and a prenyl side chain $\left[\delta_{\mathrm{H}} 3.35\left(\mathrm{H}-1^{\prime \prime \prime \prime \prime}\right), 5.24\right.$ $\left(\mathrm{H}-2^{\prime \prime \prime \prime \prime}\right), 1.66\left(\mathrm{H}-4^{\prime \prime \prime \prime \prime}\right)$, and $\left.1.68\left(\mathrm{H}-5^{\prime \prime \prime \prime \prime}\right)\right]$, and a singlet at $\delta_{\mathrm{H}} 6.17\left(\mathrm{H}-6^{\prime \prime \prime}\right)$. The placement of the two methoxy groups was established based on the HMBC correlation of $\mathrm{H}-6^{\prime \prime \prime}\left(\delta_{\mathrm{H}}\right.$ $6.17)$ with C- $4^{\prime \prime \prime}\left(\delta_{\mathrm{C}} 102.8\right), \mathrm{C}-5^{\prime \prime \prime}\left(\delta_{\mathrm{C}} 157.5\right)$, and C- $7^{\prime \prime \prime}\left(\delta_{\mathrm{C}}\right.$ $158.6)$. The prenyl group was positioned at $\mathrm{C}-8^{\prime \prime \prime}\left(\delta_{\mathrm{C}} 110.5\right)$ based on the HMBC cross-peaks of $\mathrm{H}_{2}-1^{\prime \prime \prime \prime \prime}\left(\delta_{\mathrm{H}} 3.35\right)$ to C$8^{\prime \prime \prime}\left(\delta_{\mathrm{C}} 110.5\right), \mathrm{C}-8 \mathrm{a}^{\prime \prime \prime}\left(\delta_{\mathrm{C}} 154.3\right)$, and C-7"' $\left(\delta_{\mathrm{C}} 158.6\right)$. Methoxy groups were placed at $\mathrm{C}-5^{\prime \prime \prime}\left(\delta_{\mathrm{C}} 157.5\right)$ and $\mathrm{C}-7^{\prime \prime \prime}$ $\left(\delta_{\mathrm{C}} 158.6\right)$ upon biogenetic considerations, ${ }^{24}$ as well as based on the HMBC correlations of $\mathrm{MeO}-5^{\prime \prime \prime}\left(\delta_{\mathrm{H}} 3.73\right)$ to C-5 ${ }^{\prime \prime \prime}$ $\left(\delta_{\mathrm{C}} 157.5\right)$, and of $\mathrm{MeO}-7^{\prime \prime \prime}\left(\delta_{\mathrm{H}} 3.87\right)$ to $\mathrm{C}-7^{\prime \prime \prime}\left(\delta_{\mathrm{C}} 158.6\right)$. This assignment was corroborated by the NOEs of MeO- $5^{\prime \prime \prime}$ $\left(\delta_{\mathrm{H}} 3.73\right)$ and $\mathrm{MeO}-7^{\prime \prime \prime}\left(\delta_{\mathrm{H}} 3.87\right)$ with $\mathrm{H}-6^{\prime \prime \prime}\left(\delta_{\mathrm{H}} 6.17\right)$. The C-6 $\left(\delta_{\mathrm{C}} 109.8\right)$ to C-4" ${ }^{\prime \prime}\left(\delta_{\mathrm{C}} 26.8\right)$ linkage of the flavanone and the flavan moieties was confirmed by the HMBC correlations of H-4" ${ }^{\prime \prime}\left(\delta_{\mathrm{H}} 4.66\right)$ with C-5 $\left(\delta_{\mathrm{C}} 159.3\right)$, C-6 $\left(\delta_{\mathrm{C}} 109.8\right)$, and C-7 $\left(\delta_{\mathrm{C}} 162.8\right)$, and of $\mathrm{H}_{2}-3^{\prime \prime \prime}\left(\delta_{\mathrm{H}} 2.18\right.$ and 2.31$)$ with C-6 $\left(\delta_{\mathrm{C}}\right.$ 109.8). Because of its three stereocenters at C-2, C-2 ${ }^{\prime \prime \prime}$, and C$4^{\prime \prime \prime}, 1$ has eight possible stereoisomers. The large ${ }^{3} J_{2,3}=13.1$ $\mathrm{Hz}$ indicated $\mathrm{H}-2$ to be axial, and thereby ring $\mathrm{B}$ to be equatorial. Based on the available literature on the flavanones of this genus, C-2 is expected to be $S$-configured. ${ }^{1,25}$ Similarly, the large ${ }^{3} J_{2^{\prime \prime \prime}, 3^{\prime \prime \prime}}=11.5 \mathrm{~Hz}$ suggests $\mathrm{H}-2^{\prime \prime \prime}$ to be axial, and accordingly ring $\mathrm{B}$ of the flavan moiety to be equatorially oriented. Furthermore, based on the literature, ${ }^{25} \mathrm{C}-2^{\prime \prime \prime}$ is also expected to be $S$-configured as in the flavanones and flavans of this genus. The ${ }^{3} J_{3 \mathrm{a}^{\prime \prime \prime}, 4^{\prime \prime \prime}}=2.0$ suggests the axial-equatorial or equatorial-equatorial orientation of the involved protons, whereas ${ }^{3} J_{3 \mathrm{~b}^{\prime \prime \prime}, 4^{\prime \prime \prime}}=6.0 \mathrm{~Hz}$ gauche orientation, suggesting $\mathrm{H}$ $4^{\prime \prime \prime}$ to be axially oriented and, consequently, the flavan moiety to occupy the more favorable equatorial orientation with C-4" ${ }^{\prime \prime}$ being $R$-configured. ${ }^{25}$ The NOE correlation of $\mathrm{H}-2^{\prime \prime \prime}\left(\delta_{\mathrm{H}} 5.06\right)$ and HO-7 $\left(\delta_{\mathrm{H}} 6.80\right)$ reveals the flavanone and $\mathrm{H}-2^{\prime \prime \prime}$ to be oriented in the same direction. This absolute configuration $\left(2 S, 2^{\prime \prime \prime} S, 4^{\prime \prime \prime} R\right)$, proposed on the basis of biogenetic considerations and NMR analyses was subsequently confirmed by single-crystal X-ray crystallography (Figure 1). Thus, based on the above spectroscopic data, this new compound, rhodimer (1), was characterized as $\left(2 \mathrm{~S}, 2^{\prime \prime \prime} \mathrm{S}, 4 \mathrm{R}\right)-5,7$-dihydroxy- $5^{\prime \prime \prime}, 7^{\prime \prime \prime}$-dimethoxy-8, $8^{\prime \prime \prime}$-bis(3-methylbut-2-en-1-yl)$2^{\prime}, 2^{\prime \prime \prime}$-diphenyl-[4" ${ }^{\prime \prime}, 6$-bichroman $]$-4-one.

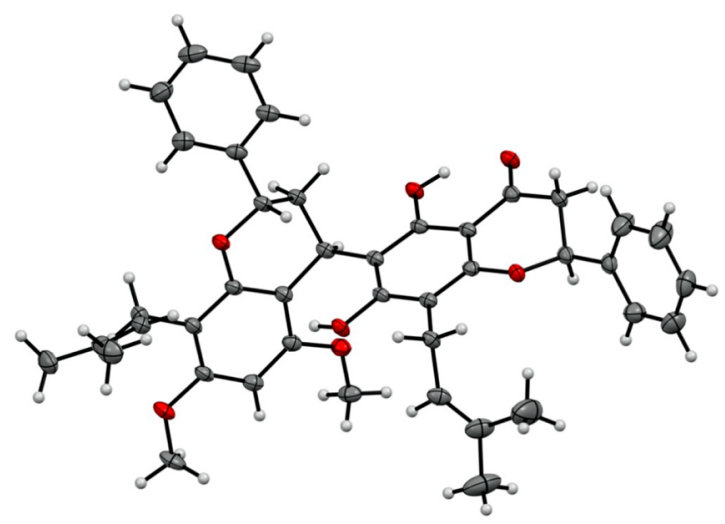

Figure 1. Single crystal X-ray structure of compound 1 with the thermal displacement parameters at $40 \%$ probability level.
Compound 2 was isolated as an oily paste and was assigned the molecular formula $\mathrm{C}_{30} \mathrm{H}_{38} \mathrm{O}_{5}$, based on HRESIMS ([ $\mathrm{M}+$ $\mathrm{H}]^{+} m / z$ 479.2493, calcd 479.2753) and NMR data analyses (see Table 2, as well as Figures S9-S14 in the Supporting Information). The ${ }^{1} \mathrm{H}$ NMR signals at $\delta_{\mathrm{H}} 5.22(\mathrm{H}-2), 1.80(\mathrm{H}-$ $\left.3_{\mathrm{ax}}\right), 2.31\left(\mathrm{H}-3_{\mathrm{eq}}\right)$, and $4.99(\mathrm{H}-4)$, along with the ${ }^{13} \mathrm{C} \mathrm{NMR}$ signals at $\delta_{\mathrm{C}} 76.1(\mathrm{C}-2), 37.0(\mathrm{C}-3)$, and 57.4 (C-4), suggested it to be a flavan-4-ol derivative, which was corroborated by the UV absorptions $\lambda_{\max }=240$ and $290 \mathrm{~nm}$. The characteristic flavan-4-ol ring $\mathrm{C}$ of $\mathbf{2}$ was confirmed by the HMBC correlations of $\mathrm{H}-4\left(\delta_{\mathrm{H}} 4.99\right)$ with C-2 $\left(\delta_{\mathrm{C}} 76.1\right), \mathrm{C}-3\left(\delta_{\mathrm{C}}\right.$ $37.0), \mathrm{C}-5\left(\delta_{\mathrm{C}} 194.7\right), \mathrm{C}-4 \mathrm{a}\left(\delta_{\mathrm{C}} 114.9\right)$, and C-8a $\left(\delta_{\mathrm{C}} 171.0\right)$, and by the COSY correlations of $\mathrm{H}-2\left(\delta_{\mathrm{H}} 5.22\right)$ with $\mathrm{CH}_{2}-3$ $\left(\delta_{\mathrm{H}} 2.31\right.$ and 1.80$)$, and of the latter with $\mathrm{H}-4\left(\delta_{\mathrm{H}} 4.99\right)$. The six methyl $\left(\mathrm{H}_{3}-4^{\prime \prime}, \mathrm{H}_{3}-4^{\prime \prime \prime}, \mathrm{H}_{3}-4^{\prime \prime \prime \prime}, \mathrm{H}_{3}-5^{\prime \prime}, \mathrm{H}_{3}-5^{\prime \prime \prime}\right.$, and $\mathrm{H}_{3}-$ $\left.5^{\prime \prime \prime}\right)$, along with the three methine signals $\left(\mathrm{H}-2^{\prime \prime}, \mathrm{H}-2^{\prime \prime \prime}\right.$, and $\left.\mathrm{H}-2^{\prime \prime \prime \prime}\right)$ were diagnostic for three prenyl groups. The NMR signals of $\mathrm{H}-2^{\prime} / 6^{\prime}, \mathrm{H}-3^{\prime} / 5^{\prime}$, and $\mathrm{H}-4^{\prime}$ (Table 2) further suggested ring $B$ to be unsubstituted and, hence, all three prenyl groups to be connected to ring $\mathrm{A}$. This ring is oxygenated at C-5 $\left(\delta_{\mathrm{C}} 194.7\right)$ and C-7 $\left(\delta_{\mathrm{C}} 206.4\right)$, with their placement having been derived from biogenetic considerations. ${ }^{24}$ An additional oxygenation is presented at C-6 $\left(\delta_{\mathrm{C}}\right.$ 83.3), which uncharacteristically is an $\mathrm{sp}^{3}$ hybridized carbon. Prenyl substitution at this carbon was confirmed by the HMBC correlation of $\mathrm{H}-2^{\prime \prime \prime \prime}\left(\delta_{\mathrm{H}} 4.99\right)$ with C-6 $\left(\delta_{\mathrm{C}} 83.3\right)$. The location of the other two prenyl groups at C- $8\left(\delta_{\mathrm{C}} 58.3\right)$, also $\mathrm{sp}^{3}$ hybridized, was established from the ${ }^{3} J \mathrm{HMBC}$ correlations of $\mathrm{H}-2^{\prime \prime}\left(\delta_{\mathrm{H}} 5.03, m\right)$ and $\mathrm{H}-2^{\prime \prime \prime}\left(\delta_{\mathrm{H}} 4.66, m\right)$ with C-8 $\left(\delta_{\mathrm{C}}\right.$ 58.3). Moreover, $\mathrm{CH}_{2}-1^{\prime \prime}\left(\delta_{\mathrm{H}} 2.60\right.$ and 2.81) and $\mathrm{CH}_{2}-1^{\prime \prime \prime}$ $\left(\delta_{\mathrm{H}} 2.46\right.$ and 2.77$)$ showed HMBC cross-peaks with C-7 $\left(\delta_{\mathrm{C}}\right.$ $206.4)$ and $\mathrm{C}-8 \mathrm{a}\left(\delta_{\mathrm{C}} 171.0\right)$, whereas $\mathrm{CH}_{2}-1^{\prime \prime \prime \prime}\left(\delta_{\mathrm{H}} 2.71\right.$ and $2.25)$ with $\mathrm{C}-5\left(\delta_{\mathrm{C}} 194.7\right)$, confirming that ring $\mathrm{A}$ is fully substituted. Based on the above data, compound 2 was characterized as 4,6-dihydroxy-6,8,8-tris(3-methylbut-2-en-1yl)-2-phenyl-2,3,4,8-tetrahydro-5H-chromene-5,7(6H)-dione. Its configurational assignment is discussed below, together with that of the structurally closely related compound 3 .

Compound 3 was isolated as an oily paste and was assigned the molecular formula $\mathrm{C}_{30} \mathrm{H}_{38} \mathrm{O}_{5}$ based on HRESIMS ([ $\mathrm{M}+$ $\mathrm{H}]^{+} m / z$ 479.2493, calcd 479.2753) and NMR data (see Table 2 , as well as Figures S16-S21 in the Supporting Information). The ${ }^{1} \mathrm{H}$ NMR signals at $\delta_{\mathrm{H}} 5.24(\mathrm{H}-2), 2.00$ and $2.30\left(\mathrm{CH}_{2}-\right.$ $3)$, and $\delta_{\mathrm{H}} 4.55(\mathrm{H}-4)$ and ${ }^{13} \mathrm{C}$ NMR signals at $\delta_{\mathrm{C}} 76.2(\mathrm{C}-2)$, 36.5 (C-3), and 59.5 (C-4) suggested 3 to be a flavan derivative with identical core structure, 4,6-dihydroxy-6,8,8tris(3-methylbut-2-en-1-yl)-2-phenyl-2,3,4,8-tetrahydro- $5 H$ chromene-5,7 $6 \mathrm{H}$ )-dione to compound 2 . The highly similar NMR data of $\mathbf{2}$ and 3 (Table 2) indicated these compounds to be stereoisomers.

Compounds $2\left([\alpha]_{\mathrm{D}}+66.1\right)$ and $3\left([\alpha]_{\mathrm{D}}+17.5\right)$ are both dextrorotatory (for details, see the Experimental Section) and, hence, are diastereomers (as enantiomers would be expected to rotate plan-polarized light in opposite directions). The large coupling constant ${ }^{3} J_{2,3 a x}(12.6 \mathrm{~Hz}$ in both 2 and 3$)$ is consistent with $\mathrm{H}-2$ being axial and ring $\mathrm{B}$ of both compounds to occupy the more favorable equatorial orientation. ${ }^{25}$ The ${ }^{3} J_{3 \mathrm{ax}, 4}(4.2 \mathrm{~Hz}$ for 2 , and $3.7 \mathrm{~Hz}$ for 3 ) and ${ }^{3} J_{3 \mathrm{eq}, 4}(1.9 \mathrm{~Hz}$ in $2,2.3 \mathrm{~Hz}$ in 3 ) are small, suggesting $\mathrm{H}-4$ to be equatorial and hence $\mathrm{HO}-4$ to be axial in both compounds, according to Pouget et al. ${ }^{26}$ This is further corroborated by the $3.4 \AA \mathrm{H}-2-\mathrm{H}-4$ interatomic distance of 3 , estimated based on the NOE cross-peak intensities using $\mathrm{H}-3 \mathrm{a} / \mathrm{b}$ as an internal reference (1.8 $\AA$; see 
Table 2. NMR Spectroscopic Data $\left(800 \mathrm{MHz}, \mathrm{CDCl}_{3}\right)$ for Rhodiflavan A (2) and Rhodiflavan B (3)

\begin{tabular}{|c|c|c|c|c|c|c|}
\hline \multirow[b]{2}{*}{ position } & \multicolumn{3}{|c|}{2} & \multicolumn{3}{|c|}{3} \\
\hline & $\delta_{\mathrm{C}}$, type & $\delta_{\mathrm{H},} \mathrm{m}(J$ in $\mathrm{Hz})$ & HMBC & $\delta_{\mathrm{C}}$, type & $\delta_{\mathrm{H},} \mathrm{m}(J$ in $\mathrm{Hz})$ & HMBC \\
\hline 2 & 76.1, $\mathrm{CH}$ & 5.22, dd $(12.6,2.3)$ & $\mathrm{C}-3, \mathrm{C}-4, \mathrm{C}-1^{\prime}, \mathrm{C}-2^{\prime}, \mathrm{C}-6^{\prime}$ & $76.2, \mathrm{CH}$ & 5.24, dd $(12.6,2.3)$ & C-3, C-4, C-1' ${ }^{\prime}, \mathrm{C}-2^{\prime}, \mathrm{C}-6^{\prime}$ \\
\hline \multirow[t]{2}{*}{3} & $37.0, \mathrm{CH}_{2}$ & $\begin{array}{l}\text { 2.31, ddd }(14.6,2.3 \text {, } \\
1.9)\end{array}$ & $C-4, C-4 a$ & $36.5, \mathrm{CH}_{2}$ & $2.30, \mathrm{dt}(14.6,2.3,2.0)$ & C-4, C-4a \\
\hline & & $\begin{array}{l}\text { 1.80, ddd }(14.6,12.6 \text {, } \\
4.2)\end{array}$ & $\mathrm{C}-1^{\prime}, \mathrm{C}-2$ & & $\begin{array}{l}2.00, \text { ddd }(14.6,12.6 \text {, } \\
3.6)\end{array}$ & $\mathrm{C}-2, \mathrm{C}-1^{\prime}$ \\
\hline 4 & $57.4, \mathrm{CH}$ & $4.99, \mathrm{~m}$ & C-2, C-3, C-5, C-4a, C-8a & $59.5, \mathrm{CH}$ & $4.55, \mathrm{dd}(2.0,3.6)$ & C-2, C-4a, C-8a, C-5 \\
\hline $4 a$ & $114.9, \mathrm{C}$ & & & $114.2, \mathrm{C}$ & & \\
\hline 5 & 194.7, C-O & & & 197.0, C-O & & \\
\hline 6 & $\begin{array}{l}\text { 83.3, } \\
\text { C-OH }\end{array}$ & & & $\stackrel{83.4,}{\mathrm{C}-\mathrm{OH}}$ & & \\
\hline 7 & $\begin{array}{l}206.4 \\
\mathrm{C}=\mathrm{O}\end{array}$ & & & $\begin{array}{l}207.2 \\
\mathrm{C}=\mathrm{O}\end{array}$ & & \\
\hline 8 & 58.3, C & & & 58.3, C & & \\
\hline $8 \mathrm{a}$ & $171.0, \mathrm{C}-\mathrm{O}$ & & & $171.0, \mathrm{C}-\mathrm{O}$ & & \\
\hline $1^{\prime}$ & $138.9, \mathrm{C}$ & & & 139.2, C & & \\
\hline $2^{\prime}, 6^{\prime}$ & $125.9, \mathrm{CH}$ & $7.40, \mathrm{~m}$ & C-2, C- $2^{\prime}, \mathrm{C}-4^{\prime}, \mathrm{C}-6^{\prime}$ & 125.9, $\mathrm{CH}$ & $7.40, \mathrm{~m}$ & $\mathrm{C}-2, \mathrm{C}-4^{\prime}, \mathrm{C}-2^{\prime}, \mathrm{C}-6^{\prime}$ \\
\hline $3^{\prime}, 5^{\prime}$ & $128.7, \mathrm{CH}$ & $7.43, \mathrm{~m}$ & $\mathrm{C}-1^{\prime}, \mathrm{C}-3^{\prime}, \mathrm{C}-5^{\prime}$ & $128.7, \mathrm{CH}$ & $7.43, \mathrm{~m}$ & $\mathrm{C}-1^{\prime}, \mathrm{C}-3^{\prime}, \mathrm{C}-5^{\prime}$ \\
\hline $4^{\prime}$ & $128.5, \mathrm{CH}$ & $7.39, \mathrm{~m}$ & $C-1^{\prime}, C-2^{\prime}, C-6^{\prime}$ & $128.5, \mathrm{CH}$ & $7.39, \mathrm{~m}$ & $\mathrm{C}-1^{\prime}, \mathrm{C}-2^{\prime}, \mathrm{C}-6^{\prime}$ \\
\hline \multirow[t]{2}{*}{$1^{\prime \prime}$} & $35.0, \mathrm{CH}_{2}$ & 2.81, dd $(14.2,9.2)$ & $\begin{array}{l}\mathrm{C}-7, \mathrm{C}-8, \mathrm{C}-8 \mathrm{a}, \mathrm{C}-2^{\prime \prime}, \mathrm{C}-3^{\prime \prime} \text {, } \\
\mathrm{C}-1^{\prime \prime \prime}\end{array}$ & $34.5, \mathrm{CH}_{2}$ & 2.89, dd $(14.2,9.8)$ & $\begin{array}{l}\mathrm{C}-7, \mathrm{C}-8, \mathrm{C}-8 \mathrm{a}, \mathrm{C}-2^{\prime \prime}, \mathrm{C}-3^{\prime \prime}, \\
\mathrm{C}-1^{\prime \prime \prime}\end{array}$ \\
\hline & & 2.60, dd $(6.3,14.2)$ & $\begin{array}{l}\mathrm{C}-7, \mathrm{C}-8, \mathrm{C}-8 \mathrm{a}, \mathrm{C}-2^{\prime \prime}, \mathrm{C}-3^{\prime \prime} \text {, } \\
\mathrm{C}-1^{\prime \prime \prime}\end{array}$ & & $2.59, \mathrm{~m}$ & $\begin{array}{l}\mathrm{C}-7, \mathrm{C}-8, \mathrm{C}-8 \mathrm{a}, \mathrm{C}-2^{\prime \prime}, \mathrm{C}-3^{\prime \prime} \text {, } \\
\mathrm{C}-1^{\prime \prime \prime}\end{array}$ \\
\hline \multirow[t]{2}{*}{$1^{\prime \prime \prime}$} & $38.5, \mathrm{CH}_{2}$ & $2.77, \mathrm{~m}$ & $\begin{array}{l}\mathrm{C}-7, \mathrm{C}-8, \mathrm{C}-8 \mathrm{a}, \mathrm{C}-2^{\prime \prime \prime}, \mathrm{C}-3^{\prime \prime \prime} \text {, } \\
\mathrm{C}-1^{\prime \prime}\end{array}$ & $38.5, \mathrm{CH}_{2}$ & $2.69, \mathrm{dd}(13.5,7.3)$ & $\begin{array}{l}\mathrm{C}-7, \mathrm{C}-8, \mathrm{C}-8 \mathrm{a}, \mathrm{C}-2^{\prime \prime \prime}, \mathrm{C}-3^{\prime \prime \prime} \text {, } \\
\mathrm{C}-1^{\prime \prime}\end{array}$ \\
\hline & & 2.46 , dd $(13.7,8.2)$ & $\begin{array}{l}\mathrm{C}-7, \mathrm{C}-8, \mathrm{C}-8 \mathrm{a}, \mathrm{C}-2^{\prime \prime \prime}, \mathrm{C}-3^{\prime \prime \prime}, \\
\mathrm{C}-1^{\prime \prime}\end{array}$ & & $2.43, \mathrm{dd}(13.5,8.7)$ & $\begin{array}{l}\mathrm{C}-7, \mathrm{C}-8, \mathrm{C}-8 \mathrm{a}, \mathrm{C}-2^{\prime \prime \prime}, \mathrm{C}-3^{\prime \prime \prime} \text {, } \\
\mathrm{C}-1^{\prime \prime}\end{array}$ \\
\hline \multirow[t]{2}{*}{$1^{\prime \prime \prime \prime}$} & $38.5, \mathrm{CH}_{2}$ & 2.71, dd $(14.9,6.3)$ & $\mathrm{C}-6, \mathrm{C}-5, \mathrm{C}-2^{\prime \prime \prime \prime}$ & $38.2, \mathrm{CH}_{2}$ & $2.63, \mathrm{dd}(14.7,8.7)$ & $\mathrm{C}-6, \mathrm{C}-5, \mathrm{C}-2^{\prime \prime \prime \prime}$ \\
\hline & & 2.25, dd $(15.0,5.7)$ & $\mathrm{C}-6, \mathrm{C}-5, \mathrm{C}-2^{\prime \prime \prime \prime}$ & & $2.18, \mathrm{~m}$ & $\mathrm{C}-6, \mathrm{C}-5, \mathrm{C}-2^{\prime \prime \prime \prime}$ \\
\hline $2^{\prime \prime}$ & $118.8, \mathrm{CH}$ & $5.03, \mathrm{~m}$ & $\mathrm{C}-1^{\prime \prime}, \mathrm{C}-4^{\prime \prime}, \mathrm{C}-5^{\prime \prime}$ & $118.8, \mathrm{CH}$ & $5.13, \mathrm{~m}$ & $\mathrm{C}-8, \mathrm{C}-1^{\prime \prime}, \mathrm{C}-4^{\prime \prime}, \mathrm{C}-5^{\prime \prime}$ \\
\hline $2^{\prime \prime \prime}$ & $117.4, \mathrm{CH}$ & $4.66, \mathrm{~m}$ & $\mathrm{C}-8, \mathrm{C}-1^{\prime \prime \prime}, \mathrm{C}-4^{\prime \prime \prime}, \mathrm{C}-5^{\prime \prime \prime}$ & $117.4, \mathrm{CH}$ & $4.71, \mathrm{~m}$ & $\mathrm{C}-8, \mathrm{C}-1^{\prime \prime \prime}, \mathrm{C}-4^{\prime \prime \prime}, \mathrm{C}-5^{\prime \prime \prime}$ \\
\hline $2^{\prime \prime \prime \prime}$ & 115.6, $\mathrm{CH}$ & $4.99, \mathrm{~m}$ & $\mathrm{C}-6, \mathrm{C}-4^{\prime \prime \prime \prime}, \mathrm{C}-5^{\prime \prime \prime \prime}$ & $115.5, \mathrm{CH}$ & $4.97, \mathrm{~m}$ & $\mathrm{C}-6, \mathrm{C}-4^{\prime \prime \prime \prime}, \mathrm{C}-5^{\prime \prime \prime \prime}$ \\
\hline $3^{\prime \prime}$ & $136.0, \mathrm{C}$ & & & 136.0, C & & \\
\hline $3^{\prime \prime \prime}$ & $136.9, \mathrm{C}$ & & & $136.9, \mathrm{C}$ & & \\
\hline $3^{\prime \prime \prime \prime}$ & $136.9, \mathrm{C}$ & & & $136.9, \mathrm{C}$ & & \\
\hline $4^{\prime \prime}$ & 26.0, $\mathrm{CH}_{3}$ & $1.64, \mathrm{~s}$ & $\mathrm{C}-1^{\prime \prime}, \mathrm{C}-2^{\prime \prime}, \mathrm{C}-5^{\prime \prime}$ & 18.0, $\mathrm{CH}_{3}$ & $1.61, \mathrm{~s}$ & $\mathrm{C}-1^{\prime \prime}, \mathrm{C}-2^{\prime \prime}, \mathrm{C}-5^{\prime \prime}$ \\
\hline $4^{\prime \prime \prime}$ & $18.1, \mathrm{CH}_{3}$ & $1.52, \mathrm{~s}$ & $\mathrm{C}-1^{\prime \prime \prime}, \mathrm{C}-2^{\prime \prime \prime}, \mathrm{C}-5^{\prime \prime \prime}$ & $17.8, \mathrm{CH}_{3}$ & $1.52, \mathrm{~s}$ & $\mathrm{C}-1^{\prime \prime \prime}, \mathrm{C}-2^{\prime \prime \prime}, \mathrm{C}-5^{\prime \prime \prime}$ \\
\hline $4^{\prime \prime \prime \prime}$ & 26.0, $\mathrm{CH}_{3}$ & $1.71, \mathrm{~s}$ & $\mathrm{C}-2^{\prime \prime \prime \prime}, \mathrm{C}-3^{\prime \prime \prime \prime}, \mathrm{C}-5^{\prime \prime \prime \prime}$ & 25.9, $\mathrm{CH}_{3}$ & $1.71, \mathrm{~s}$ & $\mathrm{C}-2^{\prime \prime \prime}, \mathrm{C}-3^{\prime \prime \prime \prime}, \mathrm{C}-5^{\prime \prime \prime \prime}$ \\
\hline $5^{\prime \prime}$ & 18.1, $\mathrm{CH}_{3}$ & $1.59, \mathrm{~s}$ & $\mathrm{C}-1^{\prime \prime}, \mathrm{C}-2^{\prime \prime}, \mathrm{C}-4^{\prime \prime \prime}$ & 26.0, $\mathrm{CH}_{3}$ & $1.68, \mathrm{~s}$ & $\mathrm{C}-1^{\prime \prime}, \mathrm{C}-2^{\prime \prime}, \mathrm{C}-4^{\prime \prime \prime}$ \\
\hline $5^{\prime \prime \prime}$ & 25.8, $\mathrm{CH}_{3}$ & $1.55, \mathrm{~s}$ & $\mathrm{C}-1^{\prime \prime}, \mathrm{C}-2^{\prime \prime}, \mathrm{C}-4^{\prime \prime \prime}$ & 25.7, $\mathrm{CH}_{3}$ & $1.56, \mathrm{~s}$ & $\mathrm{C}-1^{\prime \prime}, \mathrm{C}-2^{\prime \prime}, \mathrm{C}-4^{\prime \prime \prime}$ \\
\hline $5^{\prime \prime \prime \prime}$ & $17.9, \mathrm{CH}_{3}$ & $1.55, \mathrm{~s}$ & $\mathrm{C}-2^{\prime \prime \prime \prime}, \mathrm{C}-3^{\prime \prime \prime \prime}, \mathrm{C}-4^{\prime \prime \prime \prime}$ & $17.9, \mathrm{CH}_{3}$ & $1.54, \mathrm{~s}$ & $\mathrm{C}-2^{\prime \prime \prime \prime}, \mathrm{C}-3^{\prime \prime \prime \prime}, \mathrm{C}-4^{\prime \prime \prime \prime}$ \\
\hline
\end{tabular}

Figure S23 in the Supporting Information). This distance was computed to $2.3 \AA$ for the $\left(2 S^{*}, 4 S^{*}\right)$ diastereomer, and $3.7 \AA$ for the $\left(2 S^{*}, 4 R^{*}\right)$ configured C-ring, using Boltzmann averaging of the $\mathrm{H}-2-\mathrm{H}-3$ distances of the output conformers of Monte Carlo conformational searches (see Experimental Section for details; this distance was not measured for 2, because of signal overlap). Based on biogenetic considerations discussed for compound $\mathbf{1}$ and the NMR data, compounds $\mathbf{2}$ and 3 are C-6 epimers and possess a $(2 S, 4 R)$ absolute configuration. In order to determine their absolute configuration, their electronic circular dichroism (ECD) spectra (Figure 2) were recorded. The observed Cotton effects supported the ORD-based suggestion of the diastereomeric relationship of $\mathbf{2}$ and $\mathbf{3}$ as enantiomers are expected to show mirror-image ECD spectra. The comparison of the experimental spectra with those reported for flavan-4-ols ${ }^{11}$ did not allow configurational assignment, because of the dissimilarity of rings A of $\mathbf{2}$ and 3, compared to those of the reported compounds. Consequently, the Boltzmann-weighted ECD spectra of the possible diastereomers were calculated using

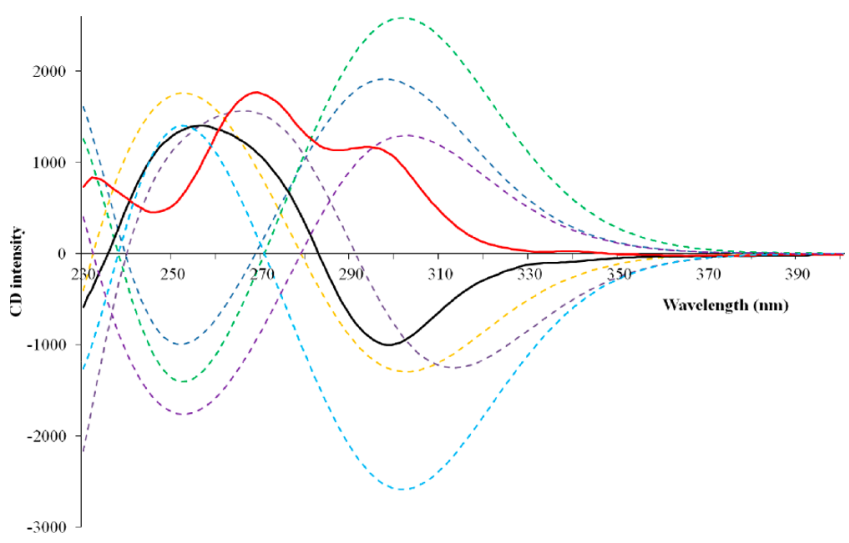

Figure 2. ECD spectra of compounds 2 (black) and 3 (red) along with the calculated spectra of the stereoisomers $[(2 S, 4 R, 6 S)$ yellow, $(2 S, 4 R, 6 R)$ blue, $(2 S, 4 S, 6 R)$ green, $(2 S, 4 S, 6 S)$ lilac, $(2 R, 4 S, 6 R)$ pink, $(2 R, 4 S, 6 S)$ light blue]. See details of the ECD calculations in the Experimental Section. 
Table 3. ${ }^{1} \mathrm{H}$ and ${ }^{13} \mathrm{C}$ NMR Spectroscopic Data (800 $\mathrm{MHz}, \mathrm{CDCl}_{3}$ ) for Rhodiflavan C (4)

\begin{tabular}{|c|c|c|c|}
\hline position & $\delta_{\mathrm{C}}$, type & $\delta_{\mathrm{H}, \mathrm{m}}(J$ in $\mathrm{Hz})$ & HМBC \\
\hline 2 & $78.8, \mathrm{CH}$ & 5.45 , dd $(12.3,2.2)$ & $\mathrm{C}-3, \mathrm{C}-4, \mathrm{C}-1^{\prime}, \mathrm{C}-2^{\prime} / \mathrm{C}-6^{\prime}$ \\
\hline \multirow[t]{2}{*}{3} & \multirow[t]{2}{*}{ 37.0, $\mathrm{CH}_{2}$} & $2.31, \mathrm{dt}(14.8,2.3)$ & C-4, C-4a \\
\hline & & 2.02, ddd $(14.9,12.3,3.5)$ & $\mathrm{C}-2, \mathrm{C}-1^{\prime}$ \\
\hline 4 & $55.6, \mathrm{CH}$ & $4.86, \mathrm{~m}$ & C-2, C-5, C-4a, C-7a \\
\hline $4 a$ & $125.9, \mathrm{C}$ & & \\
\hline 5 & $184.5, \mathrm{C}=\mathrm{O}$ & & \\
\hline 6 & $203.4 \mathrm{C}=\mathrm{O}$ & & \\
\hline 7 & 52.9, C, & & \\
\hline $7 a$ & 188.6, $\mathrm{C}-\mathrm{O}$ & & \\
\hline $1^{\prime}$ & 137.9, C & & \\
\hline $2^{\prime}, 6^{\prime}$ & $126.3, \mathrm{CH}$ & $7.40, \mathrm{~m}$ & $\mathrm{C}-2, \mathrm{C}-2^{\prime}, \mathrm{C}-4^{\prime}, \mathrm{C}-6^{\prime}$ \\
\hline $3^{\prime}, 5^{\prime}$ & $128.9, \mathrm{CH}$ & $7.46, \mathrm{~m}$ & $\mathrm{C}-1^{\prime}, \mathrm{C}-2^{\prime}, \mathrm{C}-3^{\prime}, \mathrm{C}-5^{\prime}, \mathrm{C}-6^{\prime}$ \\
\hline $4^{\prime}$ & 129.1, CH & $7.43, \mathrm{~m}$ & $\mathrm{C}-2^{\prime}, \mathrm{C}-6^{\prime}, \mathrm{C}-3^{\prime}, \mathrm{C}-5^{\prime}$ \\
\hline \multirow[t]{2}{*}{$1^{\prime \prime}$} & \multirow{2}{*}{$32.8, \mathrm{CH}_{2}$} & $2.51, \mathrm{dd}(14.3,7.2)$ & $\mathrm{C}-5, \mathrm{C}-6, \mathrm{C}-7, \mathrm{C}-7 \mathrm{a}, \mathrm{C}-2^{\prime \prime}, \mathrm{C}-3^{\prime \prime}, \mathrm{C}-1^{\prime \prime \prime}$ \\
\hline & & 2.42 , dd $(14.3,7.9)$ & $\mathrm{C}-5, \mathrm{C}-6, \mathrm{C}-7, \mathrm{C}-7 \mathrm{a}, \mathrm{C}-2^{\prime \prime}, \mathrm{C}-3^{\prime \prime}, \mathrm{C}-1^{\prime \prime \prime}$ \\
\hline \multirow[t]{2}{*}{$1^{\prime \prime \prime}$} & \multirow[t]{2}{*}{ 32.6, $\mathrm{CH}_{2}$} & $2.56, \mathrm{dd}(14.2,7.2)$ & $\mathrm{C}-5, \mathrm{C}-6, \mathrm{C}-7, \mathrm{C}-7 \mathrm{a}, \mathrm{C}-2^{\prime \prime \prime}, \mathrm{C}-3^{\prime \prime \prime}, \mathrm{C}-1^{\prime \prime}$ \\
\hline & & $2.44, \mathrm{dd}(14.2,7.9)$ & $\mathrm{C}-5, \mathrm{C}-6, \mathrm{C}-7, \mathrm{C}-7 \mathrm{a}, \mathrm{C}-1^{\prime \prime}, \mathrm{C}-2^{\prime \prime \prime}, \mathrm{C}-3^{\prime \prime \prime}$ \\
\hline $2^{\prime \prime}$ & $116.9, \mathrm{CH}$ & $4.86, \mathrm{~m}$ & $\mathrm{C}-8, \mathrm{C}-1^{\prime \prime}, \mathrm{C}-4^{\prime \prime}, \mathrm{C}-5^{\prime \prime}$ \\
\hline $2^{\prime \prime \prime}$ & $116.9, \mathrm{CH}$ & $4.86, \mathrm{~m}$ & $\mathrm{C}-8, \mathrm{C}-1^{\prime \prime \prime}, \mathrm{C}-4^{\prime \prime \prime}, \mathrm{C}-5^{\prime \prime \prime}$ \\
\hline $3^{\prime \prime}$ & 136.6, C & & \\
\hline $3^{\prime \prime \prime}$ & $136.8, \mathrm{C}$ & & \\
\hline $4^{\prime \prime}$ & 25.9, $\mathrm{CH}_{3}$ & $1.61, \mathrm{~s}$ & $\mathrm{C}-1^{\prime \prime}, \mathrm{C}-2^{\prime \prime}, \mathrm{C}-5^{\prime \prime}$ \\
\hline $4^{\prime \prime \prime}$ & 25.9, $\mathrm{CH}_{3}$ & $1.61, \mathrm{~s}$ & $\mathrm{C}-1^{\prime \prime}, \mathrm{C}-2^{\prime \prime}, \mathrm{C}-4^{\prime \prime \prime}$ \\
\hline $5^{\prime \prime}$ & $17.9, \mathrm{CH}_{3}$ & $1.57, \mathrm{~s}$ & $\mathrm{C}-1^{\prime \prime \prime}, \mathrm{C}-2^{\prime \prime \prime}, \mathrm{C}-5^{\prime \prime \prime}$ \\
\hline $5^{\prime \prime \prime}$ & $17.8, \mathrm{CH}_{3}$ & $1.56, \mathrm{~s}$ & $\mathrm{C}-1^{\prime \prime}, \mathrm{C}-2^{\prime \prime}, \mathrm{C}-4^{\prime \prime \prime}$ \\
\hline
\end{tabular}

Table 4. ${ }^{1} \mathrm{H}$ and ${ }^{13} \mathrm{C}$ NMR Spectroscopic Data (800 $\mathrm{MHz}, \mathrm{CDCl}_{3}$ ) of Compound 5

\begin{tabular}{|c|c|c|c|}
\hline position & $\delta_{\mathrm{C}}$, type & $\delta_{\mathrm{H}}, \mathrm{m}(J$ in $\mathrm{Hz})$ & HMBC \\
\hline 1 & 131.8, CH & $7.20, \mathrm{~s}$ & C-3, C-11a, C-4a, C-1' \\
\hline 2 & 121.8, C & & \\
\hline 3 & $155.8, \mathrm{C}-\mathrm{O}$ & & \\
\hline 4 & 104.0, $\mathrm{CH}$ & 6.40 & C-2, C-3, C-11b, C-4a \\
\hline $4 \mathrm{a}$ & $154.1, \mathrm{C}-\mathrm{O}$ & & \\
\hline \multirow[t]{2}{*}{6} & 69.6, $\mathrm{CH}_{2}$ & $3.95, \mathrm{~d}(11.5)$ & $C-4 a, C-6 b, C-11 a$ \\
\hline & & $4.15, \mathrm{~d}(11.5)$ & C-4a, C-6a, C-6b, C-11a \\
\hline $6 a$ & 77.2, $\mathrm{C}-\mathrm{H}$ & & \\
\hline $6 \mathrm{~b}$ & $119.0, \mathrm{C}$ & & \\
\hline 7 & 103.0, $\mathrm{CH}$ & $6.80, \mathrm{~s}$ & C-6a, C-8, C-9, C-10a \\
\hline 8 & $142.4, \mathrm{C}-\mathrm{O}$ & & \\
\hline 9 & $149.7, \mathrm{C}-\mathrm{O}$ & & \\
\hline 10 & $94.2, \mathrm{CH}$ & $6.81, \mathrm{~s}$ & C-8, C-9, C-6b, C-10a \\
\hline $10 \mathrm{a}$ & 149.7, C & & \\
\hline $11 \mathrm{a}$ & $84.8, \mathrm{C}-\mathrm{H}$ & $5.25, \mathrm{~s}$ & C-1, C-4a, C-6, C-6a, C-11b, C-10a \\
\hline $11 \mathrm{~b}$ & 112.1, C & & \\
\hline $\mathrm{OCH}_{2} \mathrm{O}-8,9$ & 101.5, $\mathrm{CH}_{2}$ & 5.91, d (1.4) 5.94, d (1.4) & C-8, C-9 \\
\hline $1^{\prime}$ & 29.2, $\mathrm{CH}_{2}$ & $3.32, \mathrm{~d}(7.2)$ & $\mathrm{C}-1, \mathrm{C}-3, \mathrm{C}-2^{\prime}, \mathrm{C}-3^{\prime}$ \\
\hline $2^{\prime}$ & $121.8, \mathrm{CH}$ & $5.31, \mathrm{~m}$ & $\mathrm{C}-4^{\prime}, \mathrm{C}-5^{\prime}, \mathrm{C}-1^{\prime}$ \\
\hline $3^{\prime}$ & $135.8, \mathrm{C}$ & & \\
\hline $4^{\prime}$ & $17.9, \mathrm{CH}_{3}$ & $1.78, \mathrm{~s}$ & $\mathrm{C}-2^{\prime}, \mathrm{C}-3^{\prime}, \mathrm{C}-5^{\prime}$ \\
\hline $5^{\prime}$ & $25.8, \mathrm{CH}_{3}$ & $1.78, \mathrm{~s}$ & $\mathrm{C}-2^{\prime}, \mathrm{C}-3^{\prime}, \mathrm{C}-4^{\prime}$ \\
\hline
\end{tabular}

the TD-DFT method (see Experimental Section for details), and compared to those obtained experimentally for $\mathbf{2}$ and $\mathbf{3}$ (Figure 2). The experimental ECD spectrum of 2 showed a negative Cotton effect at ca. $300 \mathrm{~nm}$, positive at 260, and negative at $230 \mathrm{~nm}$, best matched with the calculated spectrum of the $(2 S, 4 R, 6 S)$ isomer. Nonetheless, the mirror imaged spectrum of the $(2 S, 4 R, 6 R)$ isomer, corresponding to a $(2 R, 4 S, 6 S)$ configuration, shows similar Cotton effects. Furthermore, the calculated spectra of the $(2 S, 4 R, 6 S)$ and
$(2 S, 4 S, 6 S)$ isomers are remarkably similar, and the same goes for the other two calculated isomers. None of these show agreement with the experimentally obtained ECD spectrum of 3. Based on the above spectroscopic evidence, the new compounds rhodiflavan A (2) and B (3) are characterized as diastereomers of 4,6-dihydroxy-6,8,8-tris (3-methylbut-2-en-1yl)-2-phenyl-2,3,4,8-tetrahydro-5H-chromene-5,7(6H)-dione, with $\left(2 S^{*}, 4 R^{*}, 6 S^{*}\right)$ and $\left(2 S^{*}, 4 R^{*}, 6 R^{*}\right)$ relative configurations, respectively. 
Compound 4 was isolated as an oily paste. Its molecular formula was deduced as $\mathrm{C}_{24} \mathrm{H}_{28} \mathrm{O}_{4}$ based on HREIMS $(\mathrm{m} / z$ $[\mathrm{M}]^{+}$381.2062, calcd 381.2066) and NMR data (see Table 3, as well as Figures S23-S28 in the Supporting Information) analyses. The ${ }^{1} \mathrm{H}$ NMR signals at $\delta_{\mathrm{H}} 5.45(\mathrm{H}-2), 2.02\left(\mathrm{H}-3_{\mathrm{ax}}\right)$, $2.31\left(\mathrm{H}-3_{\text {eq }}\right)$, and $4.86(\mathrm{H}-4)$, the ${ }^{13} \mathrm{C}$ NMR signals at $\delta_{\mathrm{C}} 78.8$ (C-2), 37.0 (C-3), and 55.6 (C-4), along with the UV $\lambda_{\max }$ at 230 and $270 \mathrm{~nm}$ suggested compound 4 to have an analogous ring $\mathrm{C}$ to those of 2 and 3. Its ${ }^{1} \mathrm{H}$ NMR signals at $\delta_{\mathrm{H}} 7.4(\mathrm{H}-$ $\left.2^{\prime} / 6^{\prime}\right), 7.46\left(\mathrm{H}-3^{\prime} / 5^{\prime}\right)$, and $7.43\left(\mathrm{H}-4^{\prime}\right)$ indicated ring $\mathrm{B}$ to be unsubstituted. The lack of aromatic protons for ring $\mathrm{A}$ suggested it to be fully substituted. As rings $\mathrm{B}$ and $\mathrm{C}$ were fully assigned, the carbonyl groups at $\delta_{\mathrm{C}} 184.5$ (C-5) and 203.4 (C-6), and the two prenyl groups (see Table 3 for signals $1^{\prime \prime}-$ $5^{\prime \prime}$, and $\left.1^{\prime \prime \prime}-5^{\prime \prime}\right)$ could only be related to ring A. The HMBC correlations of $\mathrm{H}-2^{\prime \prime}\left(\delta_{\mathrm{H}} 4.86\right)$ and $\mathrm{H}-2^{\prime \prime \prime}\left(\delta_{\mathrm{H}} 4.86\right)$ with C-7 $\left(\delta_{\mathrm{C}} 52.9\right)$ revealed the two prenyl groups to be both attached to C-7. Both methylene groups $\mathrm{CH}_{2}-2^{\prime \prime}\left(\delta_{\mathrm{H}} 4.86\right)$ and $\mathrm{CH}_{2}-$ $2^{\prime \prime \prime}\left(\delta_{\mathrm{H}} 4.86\right)$ showed HMBC correlation to $\delta_{\mathrm{C}} 188.6(\mathrm{C}-7 \mathrm{a})$, $\delta_{\mathrm{C}} 203.4(\mathrm{C}-6)$, and $\delta_{\mathrm{C}} 52.9(\mathrm{C}-7)$, indicating the placement of the two carbonyl groups at C-5 and C-6, which, being a fivemembered ring, is unique for the genus Tephrosia. The difference in the chemical shifts of C-5 $\left(\delta_{\mathrm{C}} 184.5\right)$ and C-6 $\left(\delta_{\mathrm{C}}\right.$ $203.4)$ is rationalized by the strong intramolecular hydrogen bond of HO-4 and the oxygen of the C-5 carbonyl group, and C-5 has strong conjugation with C-7a through the $4 a$ (7a) double bond. The observed mass and NMR data (vide supra), the UV spectrum only showing a benzenoid band, and the $\mathrm{HMBC}$ of $\mathrm{H}-4\left(\delta_{\mathrm{H}} 4.86\right)$ with C-5 $\left(\delta_{\mathrm{C}} 184.5\right)$ corroborate the proposed structure. It is optically active $\left([\alpha]_{\mathrm{D}}+90(c 0.001\right.$, $\mathrm{CH}_{2} \mathrm{Cl}_{2}$ ), and its ECD spectrum (see Figure $\mathrm{S} 30$ in the Supporting Information) shows a positive Cotton effect at $\lambda$ $300-340 \mathrm{~nm}$ and a negative one at $275 \mathrm{~nm}$. Based on the similarity of the NMR data $(\delta$, $J$, Table 3$)$ of $\mathrm{H}-2, \mathrm{H}-3$, and $\mathrm{H}-$ 4 (ring C) of 4 to those of compounds 2 and 3 (Table 2), compound 4 was assigned the $\left(2 S^{*}, 4 R^{*}\right)$ relative configuration. Therefore, based on the above spectroscopic data, this unique compound, rhodiflavan $\mathrm{C}$ (4), was characterized as $\left(2 S^{*}, 4 R^{*}\right)$-4-hydroxy-7,7-bis(3-methylbut-2-en-1-yl)-2-phenyl2,3,4,7-tetrahydrocyclopenta[b]pyran-5,6-dione.

Compound 5 was isolated as an amorphous solid and was assigned the molecular formula $\mathrm{C}_{21} \mathrm{H}_{20} \mathrm{O}_{6}$, based on HRESIMS $\left([\mathrm{M}+\mathrm{H}]^{+} m / z\right.$ obs 369.1367$)$ and NMR data (see Table 4, as well as Figures S31-S36 in the Supporting Information) analyses. It showed characteristic UV $\left(\lambda_{\max } 230,190,310\right.$, and $350 \mathrm{~nm}),{ }^{1} \mathrm{H}$ NMR $\left[\delta_{\mathrm{H}} 3.95\right.$ and $4.15\left(\mathrm{CH}_{2}-6\right), 5.25(\mathrm{H}-$ 11a) $]$, and ${ }^{13} \mathrm{C}$ NMR data $\left[\delta_{\mathrm{C}} 69.6\right.$ (C-6), 77.2 (C-6a), 84.8 (C-11a) for a 6a-hydroxypterocarpan skeleton. The NMR data further indicated the presence of a prenyl group $\left[\delta_{\mathrm{H}} 3.32\left(\mathrm{H}_{2^{-}}\right.\right.$ $\left.1^{\prime}\right), 5.31\left(\mathrm{H}-2^{\prime}\right), 1.78\left(\mathrm{H}_{3}-4^{\prime}\right.$ and $\left.\mathrm{H}_{3}-5^{\prime}\right)$, and $\delta_{\mathrm{C}} 29.2\left(\mathrm{C}-1^{\prime}\right)$ $\left.121.8\left(\mathrm{C}-2^{\prime}\right), 135.8\left(\mathrm{C}-3^{\prime}\right), 17.9\left(\mathrm{C}-4^{\prime}\right), 25.8\left(\mathrm{C}-5^{\prime}\right)\right]$ connected to $\mathrm{C}-2$, as revealed by HMBC cross-peaks of $\mathrm{H}-1$ $\left(\delta_{\mathrm{H}} 7.20\right)$ with C-1' $\left(\delta_{\mathrm{C}} 29.2\right)$, and of $\mathrm{CH}_{2}-1^{\prime}\left(\delta_{\mathrm{H}} 3.32\right)$ with C-1 $\left(\delta_{\mathrm{C}} 131.8\right)$ and C-3 $\left(\delta_{\mathrm{C}} 155.8\right)$. The position of the prenyl group was confirmed by the NOE correlation between $\mathrm{H}-1\left(\delta_{\mathrm{H}}\right.$ 7.20) and $\mathrm{CH}_{2}-1^{\prime}\left(\delta_{\mathrm{H}} 3.32\right)$. Hence, ring $\mathrm{A}$ is substituted with a prenyl group at $\mathrm{C}-2$ and a hydroxy group at $\mathrm{C}-3$. Its $\mathrm{C}-1$ and C-4 are unsubstituted as evidenced by the two singlets in its ${ }^{1} \mathrm{H}$ NMR data. In ring $\mathrm{D}$, the NMR data (Table 4) indicated the presence of an 8, 9-methylenedioxy group $\left(\delta_{\mathrm{H}} 5.91\right.$ and 5.94; $\left.\delta_{\mathrm{C}} 101.5\right)$ and two $p$-substituted aromatic protons at $\delta_{\mathrm{H}} 6.80$ $(\mathrm{H}-7)$ and $\delta_{\mathrm{H}} 6.81$ (H-10). The NMR spectra of this compound showed resemblance to those of previously published pterocarpans. ${ }^{7,27-29}$ The absolute configuration at the $\mathrm{B} / \mathrm{C}$-ring junction was determined as $(6 \mathrm{aS}, 12 \mathrm{aS})$, based on the ECD spectrum (see Figure S34 in the Supporting Information), which exhibited a positive and negative Cotton effect at $309 \mathrm{~nm}$ and $241 \mathrm{~nm}$, respectively. ${ }^{30}$ Based on the above spectroscopic data, this new compound, rhodacarpin (5), was characterized as $(6 \mathrm{a} S, 12 \mathrm{a} S)$-2-(3-methylbut-2-en-1yl) $-6 H$ - $[1,3]$ dioxolo $\left[4^{\prime}, 5^{\prime}: 5,6\right]$ benzofuro $[3,2-c]$ chromene-3,6a$(12 \mathrm{a} H)$-diol.

The crude extract of the roots of $T$. rhodesica and the isolated compounds were tested for antiplasmodial activity using a previously established protocol. ${ }^{31}$ The crude extract gave $100 \%$ growth inhibition of the chloroquine-sensitive (3D7) strain of Plasmodium falciparum at $10 \mu \mathrm{g} / \mathrm{mL}$ concentration, whereas the isolated compounds $2,3,4,5,9$, and 15 showed moderate activities (Table 5). Among the tested compounds, 3 showed the highest activity $\left(\mathrm{IC}_{50}=5.7 \pm\right.$ $1.9 \mu \mathrm{M}) .^{32}$

Table 5. In Vitro Antiplasmodial Activities $\left(\mathrm{IC}_{50}\right)$ of Isolated Compounds against 3D7 Strains of $P$. falciparum $^{a}$

\begin{tabular}{lll}
\multicolumn{1}{c}{ sample } & \multicolumn{1}{c}{$\mathrm{IC}_{50}, \mu \mathrm{M}($ Pf3D7) } & $\mathrm{LD}_{50}(\mathrm{HEK}-293)$ \\
$\begin{array}{l}\text { T. rhodesica (roots) crude } \\
\text { extract }\end{array}$ & $\begin{array}{c}100 \% \text { active at } \\
10 \mu \mathrm{g} / \mathrm{mL}\end{array}$ \\
Rhodiflavan A (2) & $7.3 \pm 1.8$ & $71 \%$ at $40 \mu \mathrm{M}$ \\
Rhodiflavan B (3) & $5.7 \pm 1.9$ & $34 \%$ at $40 \mu \mathrm{M}$ \\
Rhodiflavan C (4) & $7.0 \pm 2.4$ & $101 \%$ at $40 \mu \mathrm{M}$ \\
Rhodacarpin (5) & $10.2 \pm 0.2$ & $27 \%$ at $40 \mu \mathrm{M}$ \\
Quercetol B (9) & $7.4 \pm 0.3$ & $87 \%$ at $40 \mu \mathrm{M}$ \\
6-Hydroxyrotenone (15) & $8.6 \pm 2.6$ & $10.9 \mu \mathrm{M}$ \\
Tephrowatsin A (21) & $14.5 \pm 0.7$ & \\
Chloroquine & 0.0047 & \\
Artesunate & 0.00067 &
\end{tabular}

${ }^{a}$ Compounds were tested independently either two or three times against Pf3D7. All compounds were tested in a single experiment against HEK 293 mammalian cells $\left(\mathrm{LD}_{50}\right)$.

In conclusion, five new compounds-rhodimer (1), rhodiflavan A (2), rhodiflavan B (3), rhodiflavan C (4), and rhodacarpin (5)-were isolated from the roots of Tephrosia rhodesica, along with 16 known natural products. Rohodimer is an unusual flavanone-flavan dimer, whereas rhodiflavan $\mathrm{C}$ has a five-membered A-ring, which is unprecedented for this genus. The crude root extract and several of its isolated constituents showed activities against the chloroquine-sensitive (3D7) strain of Plasmodium falciparum.

\section{EXPERIMENTAL SECTION}

General Experimental Procedures. Optical rotations were measured on a PerkinElmer 341-LC system, whereas ECD experiments were performed on a Jasco Model J-715 spectropolarimeter. UV spectra were recorded on a Specord S600 (Analytik Jena AG) spectrophotometer. Melting points were obtained on a Büchi Melting Point B-545 Switzerland apparatus; NMR spectra were acquired on a Bruker Avance III HD $800 \mathrm{MHz}$ NMR spectrometer equipped with a TCI cryogenic probe and were processed with the MestReNova 10.0 software, using the solvent residual signal $\left(\mathrm{CDCl}_{3} \delta_{\mathrm{H}} 7.26 ; \delta_{\mathrm{C}} 77.16\right)$ as chemical shift reference. LC-ESIMS data were obtained on a Micromass GC-TOF micro mass spectrometer (Micromass, Wythenshawe, Waters, Inc., U.K.), using direct inlet, and $70 \mathrm{eV}$ ionization voltage. TLC was performed on Merck precoated silica gel 60 F254 plates. Column chromatography was run on silica gel 60 (70-230 mesh). Gel filtration was done on Sephadex LH-20. Preparative HPLC was performed on a Waters 600E instrument using the 
Chromulan (Pikron, Ltd.) software and an $\mathrm{RP} \mathrm{C}_{8}$ Kromasil $(250 \mathrm{~mm}$ $\times 55 \mathrm{~mm}$ ) column eluting with repeated $\mathrm{CH}_{3} \mathrm{OH}-\mathrm{H}_{2} \mathrm{O}$ (5 to 95) gradients. Single-crystal X-ray data of $\mathbf{1}$ were collected at $120 \mathrm{~K}$ on an Agilent SuperNova dual wavelength diffractometer with a microfocus $\mathrm{X}$-ray source and multilayer optics monochromatized $\mathrm{Cu} \mathrm{K} \alpha(\lambda=$ $1.54184 \AA$ ) radiation.

Plant Material. The roots of Tephrosia rhodesica were collected in April 2015 from Kilungu Hills in Makueni County, Kenya. The plant specimen was identified by Mr. Patrick C. Mutiso of the School of Biological Sciences, the University of Nairobi. A voucher specimen (Mutiso-842/April 2015) was deposited at the University Herbarium of the University of Nairobi, Kenya.

Extraction and Isolation. The air-dried roots $(2 \mathrm{~kg})$ of $T$. rhodesica were ground and extracted with $\mathrm{CH}_{2} \mathrm{Cl}_{2}-\mathrm{CH}_{3} \mathrm{OH}(1: 1)(3$ $\times 2 \mathrm{~L}$ ) by percolation at room temperature to yield $70 \mathrm{~g}$ of a dark brown paste after evaporation of the solvent. A portion of the extract (37 g) was subjected to column chromatography over silica gel (400 g) eluting with a mixture of iso-hexane containing increasing amounts of EtOAc. The fraction eluted with $1 \%$ EtOAc in iso-hexane was purified by preparative $\mathrm{HPLC}\left(\mathrm{CH}_{3} \mathrm{OH}-\mathrm{H}_{2} \mathrm{O}\right.$ gradient elution) to give tephrowatsin $\mathrm{B}(6,20 \mathrm{mg}){ }^{8}$ The fraction eluted with $3 \%$ EtOAc in iso-hexane was purified by column chromatography on a Sephadex LH-20 column $\left(\mathrm{CH}_{2} \mathrm{Cl}_{2}-\mathrm{CH}_{3} \mathrm{OH} ; 1: 1\right)$ to give tephrinone $(7,200$ $\mathrm{mg})^{9}$ and glabranin $(8,100 \mathrm{mg}){ }^{10}$ The fractions eluted with $5 \%$ EtOAc in iso-hexane were purified by column chromatography on a Sephadex LH-20 column $\left(\mathrm{CH}_{2} \mathrm{Cl}_{2}-\mathrm{CH}_{3} \mathrm{OH} ; 1: 1\right)$, followed by recrystallization from $\mathrm{CH}_{2} \mathrm{Cl}_{2}-\mathrm{CH}_{3} \mathrm{OH}$ (1:1) to afford rhodimer $(1,15 \mathrm{mg})$. The fractions eluted with 6\% EtOAc in iso-hexane were subjected to column chromatography on a Sephadex LH-20 $\left(\mathrm{CH}_{2} \mathrm{Cl}_{2}-\mathrm{CH}_{3} \mathrm{OH}(1: 1)\right)$ system to give quercetol $\mathrm{B}(9,300$ $\mathrm{mg}) .{ }^{11}$ The fractions eluted with $7 \%$ EtOAc in iso-hexane were purified by column chromatography on a Sephadex LH-20 column $\left(\mathrm{CH}_{2} \mathrm{Cl}_{2}-\mathrm{CH}_{3} \mathrm{OH}(1: 1)\right)$ to give maackiain $(10,20 \mathrm{mg}),{ }^{12}$ 6ahydroxymaackiain $(11,10 \mathrm{mg}),{ }^{13}$ and pisatin $(12,5 \mathrm{mg}){ }^{14}$ The fractions eluted with $8 \%$ EtOAc in iso-hexane were subjected to column chromatography on a silica gel $(200 \mathrm{~g})$ with $3 \%$ EtOAc in isohexane to give tephrowatsin A $(\mathbf{2 1}, 10 \mathrm{mg}),{ }^{8}$ with $5 \%$ EtOAc in isohexane to give rhodiflavan A $(2,50 \mathrm{mg})$, and with $10 \% \mathrm{EtOAc}$ in isohexane to provide rhodiflavan $B(3,20 \mathrm{mg})$ and rhodiflavan $\mathrm{C}(4,15$ $\mathrm{mg})$, which were further purified by preparative HPLC $\left(\mathrm{CH}_{3} \mathrm{OH}-\right.$ $\mathrm{H}_{2} \mathrm{O}$ gradient elution). The fractions eluted with 9\%-10\% EtOAc in iso-hexane were combined and purified by column chromatography on a Sephadex LH-20 column $\left(\mathrm{CH}_{2} \mathrm{Cl}_{2}-\mathrm{CH}_{3} \mathrm{OH} ; 1: 1\right)$, and subsequently by preparative $\mathrm{HPLC}\left(\mathrm{CH}_{3} \mathrm{OH}-\mathrm{H}_{2} \mathrm{O}\right.$ gradient elution) to give tephrosin $(13,10 \mathrm{mg}),{ }^{15}$ rotenone $(14,15 \mathrm{mg}){ }^{15}$ 6hydroxyrotenone $(15,10 \mathrm{mg}),{ }^{16} 12$ a-hydroxyrotenone $(15,10 \mathrm{mg}),{ }^{17}$ rhodacarpin $(5,10 \mathrm{mg})$, hildecarpin $(17,10 \mathrm{mg}){ }^{18}$ and 3-hydroxy-2methoxy-8-9-methylenedioxypterocarpene $(18,15 \mathrm{mg}) .{ }^{19}$ The fractions eluted with $12 \% \mathrm{EtOAc}$ in iso-hexane gave isoliquirtigenin $(19,15 \mathrm{mg}),{ }^{20}$ and those eluted with $20 \%$ EtOAc in iso-hexane gave D-pinitol $(20,900 \mathrm{mg}) .^{21}$

Rhodimer (1). White crystals $\left(\mathrm{CH}_{2} \mathrm{Cl}_{2}-\mathrm{CH}_{3} \mathrm{OH} ; 1: 1\right) ;[\alpha]_{\mathrm{D}}{ }^{20}$ +13.4 (c 0.001, $\left.\mathrm{CH}_{3} \mathrm{OH}\right)$; UV $\left(\mathrm{CH}_{2} \mathrm{Cl}_{2}\right) \lambda_{\max }(\log \varepsilon) 230$ (4.05), 290 (4.25), and $350(4.30) \mathrm{nm}$; ECD $\left(c 0.05, \mathrm{CH}_{3} \mathrm{OH}\right) \lambda_{\max }(\Delta \varepsilon)$ $314(10.16), 292(-40.36), 249(-23.33), 235$ (50.08), 220 (-56.36), $212(62.23) ;{ }^{1} \mathrm{H}$ and ${ }^{13} \mathrm{C}$ NMR, see Table 1 ; HREIMS $m / z 660.3095[\mathrm{M}]^{+}$(calcd for $\left.\mathrm{C}_{42} \mathrm{H}_{44} \mathrm{O}_{7}, 660.3087\right)$.

Rhodiflavan $A$ (2). Yellow oily paste; $[\alpha]_{\mathrm{D}}{ }^{20}+17.5$ (c 0.001, $\left.\mathrm{CH}_{2} \mathrm{Cl}_{2}\right)$; UV $\left(\mathrm{CH}_{2} \mathrm{Cl}_{2}\right) \lambda_{\max }(\log \varepsilon) 240(3.05)$ and $290(3.40) \mathrm{nm}$; $\mathrm{ECD}\left(c 0.05, \mathrm{CH}_{3} \mathrm{OH}\right) \lambda_{\max }(\Delta \varepsilon) 300$ (-92.25), 257 (85.45), 243 $(-119.45) ;{ }^{1} \mathrm{H}$ and ${ }^{13} \mathrm{C}$ NMR, see Table 2; HRESIMS $m / z 479.2493$ $[\mathrm{M}+\mathrm{H}]^{+}$(calcd for $\mathrm{C}_{30} \mathrm{H}_{38} \mathrm{O}_{5}, 479.2753$ ).

Rhodiflavan $B$ (3). Yellow oily paste; $[\alpha]_{\mathrm{D}}{ }^{20}+66.1$ (c 0.001, $\left.\mathrm{CH}_{2} \mathrm{Cl}_{2}\right)$; UV $\left(\mathrm{CH}_{2} \mathrm{Cl}_{2}\right) \lambda_{\max }(\log \varepsilon) 230(3.08)$ and $280(3.20) \mathrm{nm}$; $\mathrm{ECD}\left(c 0.05, \mathrm{CH}_{3} \mathrm{OH}\right) \lambda_{\max }(\Delta \varepsilon) 301$ (199.73), 257 (-130.29), 225 (65.0); ${ }^{1} \mathrm{H}$ and ${ }^{13} \mathrm{C}$ NMR, see Table 2; HRESIMS $m / z$ 479.2493 [M $+\mathrm{H}]^{+}$(calcd for $\mathrm{C}_{30} \mathrm{H}_{38} \mathrm{O}_{5}$, 479.2753).

Rhodiflavan $C$ (4). Yellow oily paste. $[\alpha]_{\mathrm{D}}{ }^{20}+90.0$ (c 0.001, $\left.\mathrm{CH}_{2} \mathrm{Cl}_{2}\right)$; UV $\left(\mathrm{CH}_{2} \mathrm{Cl}_{2}\right) \lambda_{\max }(\log \varepsilon) 230(3.20)$ and $270(3.08) \mathrm{nm}$; $\mathrm{ECD}\left(c 0.05, \mathrm{CH}_{3} \mathrm{OH}\right) \lambda_{\max }(\Delta \varepsilon) 316$ (89.0), 284 (-32.21), 245
(91.80), 226 (-26.26), 218 (70.0), 210 (80.5); ${ }^{1} \mathrm{H}$ and ${ }^{13} \mathrm{C} \mathrm{NMR}$, see Table 3; HREIMS $m / z$ 381.2062 [M] ${ }^{+}$(calcd for $\mathrm{C}_{24} \mathrm{H}_{28} \mathrm{O}_{4}$, 381.2066).

Rhodacarpin (5). White amorphous solid; UV $\left(\mathrm{CH}_{2} \mathrm{Cl}_{2}\right) \lambda_{\max }$ (log ع) 230 (3.04), 290 (3.45), 310 (3.42) and 350 (3.10) nm; ECD (c $\left.0.05, \mathrm{CH}_{3} \mathrm{OH}\right) \lambda_{\max }(\Delta \varepsilon) 309$ (12.0), 241 (-82.1), 220 (7.0), 205 $(-80) ;{ }^{1} \mathrm{H}$ and ${ }^{13} \mathrm{C}$ NMR, see Table 4; HRESIMS $m / z$ 369.1367 [M $+\mathrm{H}]^{+}$(calcd for $\mathrm{C}_{21} \mathrm{H}_{20} \mathrm{O}_{6}, 369.1338$ ).

Plasmodium falciparum Culture. In vitro parasite culture of the P. falciparum strain 3D7 was maintained in RPMI with $10 \mathrm{mM}$ Hepes (Life Technologies), $50 \mu \mathrm{g} / \mathrm{mL}$ hypoxanthine (Sigma) and 5\% human serum from male $\mathrm{AB}$ plasma and $2.5 \mathrm{mg} / \mathrm{mL}$ AlbuMAX II (Life Technologies). Human $0^{+}$erythrocytes were obtained from the Australian Red Cross Blood Service (Agreement No. 13-04QLD-09; 17-06QLD-16). The parasites were maintained at $2 \%-8 \%$ parasitaemia $(\% \mathrm{P})$ at $5 \%$ hematocrit $(\% \mathrm{H})$, and incubated at $37{ }^{\circ} \mathrm{C}, 5 \%$ $\mathrm{CO}_{2}, 5 \% \mathrm{O}_{2}, 90 \% \mathrm{~N}_{2}$ and $95 \%$ humidity.

Plasmodium falciparum Growth Inhibition Assay. A wellestablished asexual $P$. falciparum imaging assay was used to determine parasite growth inhibition according to the procedure described by Duffy and Avery. ${ }^{31}$

Computation. A low-energy conformation library of postulated compounds 2 and 3 were generated using Macromodel as implemented in the Macromodel v12.1 Schrödinger suite by performing careful Monte Carlo conformational analysis using MMFF force fields, each with the GB/SA solvation models $\mathrm{CHCl}_{3}$ and $\mathrm{H}_{2} \mathrm{O}$. Elimination of redundant conformations was performed by comparison of heavy atom coordinates applying an RMSD cutoff set to $2.0 \AA$. Next, all unique conformers were optimized at the B3LYP/6$311++\mathrm{G}(3 \mathrm{df}, 2 \mathrm{pd})$ level of theory. The conformers having a Boltzmann weight, calculated using $\Delta H^{\circ}$, above $1 \%$ were selected for subsequent TD-DFT calculations at the same level of theory using 50 singlet excited states. To obtain the Boltzmann-weighted ECD spectrum, the individual spectra were line-broadened using a Gaussian band shape $(\sigma=0.3 \mathrm{eV})$. All (TD-)DFT calculations were performed using Gaussian 16, Revision A.03. ${ }^{33}$ The solvent was taken into account by using the integral equation formalism model (IEFPCM) as implemented in Gaussian 16 and the dielectric constant for methanol $(\varepsilon=32.613)$. To compensate for the typical underestimation of the transition energies, a $20 \mathrm{~nm}$ blue shift was applied on the computed ECD spectra for comparison with the experimental one. ${ }^{34}$

\section{ASSOCIATED CONTENT}

\section{SI Supporting Information}

CCDC 1987379 has been deposited with the Cambridge Crystallographic Data Centre. Copies of the data can be obtained, free of charge, on application to the Director, CCDC, 12 Union Road, Cambridge CB2 1EZ, U.K. (fax: +44(0)1223-336033 or e-mail: deposit@ccdc.cam.ac.uk). The Supporting Information is available free of charge at https:// pubs.acs.org/doi/10.1021/acs.jnatprod.0c00245.

Original MS and NMR spectra for all compounds, along with the corresponding NMReDATA ${ }^{35}$ for the new compounds 1-5; the X-ray structure of 1 (details are freely available on Zenodo at https://10.5281/zenodo. 3679345); NMR and MS data for compounds (1-21) (PDF)

$$
\text { Crystallographic data for } \mathbf{1} \text { (CIF) }
$$

\section{AUTHOR INFORMATION}

\section{Corresponding Authors}

Máté Erdélyi - Department of Chemistry-BMC, Uppsala University, SE-751 23 Uppsala, Sweden; Department of Chemistry and Molecular Biology, University of Gothenburg, SE-412 96 Gothenburg, Sweden; 다이.org/0000-0003- 
0359-5970; Phone: +46-72-9999166; Email: mate.erdelyi@ kemi.uu.se

Abiy Yenesew - Department of Chemistry, University of Nairobi, 30197-00100 Nairobi, Kenya; Phone: +254 733832 576; Email: ayenesew@uonbi.ac.ke

\section{Authors}

Yoseph Atilaw - Department of Chemistry, University of Nairobi, 30197-00100 Nairobi, Kenya; Department of Chemistry-BMC, Uppsala University, SE-751 23 Uppsala, Sweden

Lois Muiva-Mutisya - Department of Chemistry, University of Nairobi, 30197-00100 Nairobi, Kenya

Jonathan Bogaerts - Department of Chemistry, University of Antwerp, B-2020 Antwerp, Belgium

Sandra Duffy - Discovery Biology, Griffith Institute for Drug Discovery, Griffith University, Nathan, Qld 4111, Australia

Arto Valkonen - University of Jyvaskyla, Department of Chemistry, FI-40014 Jyväskylä, Finland; 이이.org/00000003-2806-3807

Matthias Heydenreich - Institut für Chemie, Universität Potsdam, D-14476 Potsdam, Germany

Vicky M. Avery - Discovery Biology, Griffith Institute for Drug Discovery, Griffith University, Nathan, Qld 4111, Australia

Kari Rissanen - University of Jyvaskyla, Department of Chemistry, FI-40014 Jyväskylä, Finland; (corcid.org/00000002-7282-8419

Complete contact information is available at: https://pubs.acs.org/10.1021/acs.jnatprod.0c00245

\section{Notes}

The authors declare no competing financial interest.

\section{ACKNOWLEDGMENTS}

Y.A. is grateful to the German Academic Exchange Services (DAAD) for a scholarship that was offered through the Natural Products Research Network for Eastern and Central Africa (NAPRECA). The Swedish Research Council (Swedish Research Links, NOs. 2012-6124 and 2019-03715), the International Science Program (ISP Sweden, Grant No. KEN-02), and the Australian Research Council (Grant No. LP120200557 to V.M.A.) are gratefully acknowledged for financial support. We thank the Australian Red Cross Blood Service for the provision of human blood. Jonathan Bogaerts thanks the Research foundation Flanders (FWO-Vlaanderen) for the appointment of a predoctoral scholarship (No. $1198318 \mathrm{~N}$ ) and acknowledges the Flemisch Supercomputing Centre (VSC) for providing computational resources and support. The Academy of Finland (Grant No. 314343 to A.V.) is also gratefully acknowledged for funding. The Swedish NMR Centre is acknowledged for access to an $800 \mathrm{MHz}$ spectrometer.

\section{REFERENCES}

(1) Chen, Y.; Yan, T.; Gao, C.; Cao, W.; Huang, R. Molecules 2014, $19,1432-1458$.

(2) Muiva, L. M.; Yenesew, A.; Derese, S.; Heydenreich, M.; Peter, M. G.; Akala, H. M.; Eyase, F.; Waters, N. C.; Mutai, C.; Keriko, J. M.; Walsh, D. Phytochem. Lett. 2009, 2, 99-102.

(3) Juma, W. P.; Akala, H. M.; Eyase, F. L.; Muiva, L. M.; Heydenreich, M.; Okalebo, F. A.; Gitu, P. M.; Peter, M. G.; Walsh, D. S.; Imbuga, M.; Yenesew, A. Phytochem. Lett. 2011, 4, 176-178.
(4) Touqeer, S.; Saeed, M. A.; Ajaib, M. Phytopharmacology 2013, 4, 598-637.

(5) Muiva-Mutisya, L.; Macharia, B.; Heydenreich, M.; Koch, A.; Akala, H. M.; Derese, S.; Omosa, L. K.; Yusuf, A. O.; Kamau, E.; Yenesew, A. Phytochem. Lett. 2014, 10, 179-183.

(6) Atilaw, Y.; Muiva-Mutisya, L.; Ndakala, A.; Akala, H. M.; Yeda, R.; Wu, Y. J.; Coghi, P.; Wong, V. K. W.; Erdélyi, M.; Yenesew, A. Molecules 2017, 22, 1514.

(7) Atilaw, Y.; Duffy, S.; Heydenreich, M.; Muiva-Mutisya, L.; Avery, V. M.; Erdélyi, M.; Yenesew, A. Molecules 2017, 22, 318.

(8) Gomez, F.; Quijano, L.; Calderon, J. S.; Rodriquez, C.; Rios, T. Phytochemistry 1985, 24, 1057-1059.

(9) Dagne, E.; Mammo, W.; Sterner, O. Phytochemistry 1992, 31, $3662-3663$

(10) Céspedes, C. L.; Achnine, L.; Lotina-Hennsen, B.; Salazar, J. R.; Gómez-Garibay, F.; Calderón, J. S. Pestic. Biochem. Physiol. 2001, 69, $63-76$

(11) Gomez-Garibay, F.; Quijano, L.; Calderon, J. S.; Morales, S.; Rios, T. Phytochemistry 1988, 27, 2971-2973.

(12) Mizuno, M.; Tanaka, T.; Katsuragawa, M.; Saito, H.; Iinuma, M. J. Nat. Prod. 1990, 53, 498-499.

(13) Bilton, J. N.; Debnam, J. R.; Smith, I. M. Phytochemistry 1976, $15,1411-1412$

(14) Kobayashi, A.; Akiyama, K.; Kawazu, K. Phytochemistry 1992, $32,77-78$.

(15) Stevenson, P. C.; Kite, G. C.; Lewis, G. P.; Forest, F.; Nyirenda, S. P.; Belmain, S. R.; Sileshi, G. W.; Veitch, N. C. Phytochemistry 2012, 78, 135-146.

(16) Krupadanam, G. L. D.; Srimannarayana, G.; Rao, N. V. S. Indian J. Chem., Sect. B 1978, 16B, 770-772.

(17) Oberholzer, M. E.; Rall, G. J. H.; Roux, D. G. Phytochemistry 1976, 15, 1283-1284

(18) Lwande, W.; Bentley, M. D.; Macfoy, C.; Lugemwa, F. N.; Hassanali, A.; Nyandat, E. Phytochemistry 1987, 26, 2425-2426.

(19) Lwande, W.; Bentley, M. D.; Hassanali, A. Int. J. Trop. Insect Sci. 1986, 7, 501-503.

(20) Takahashi, T.; Takasuka, N.; Iigo, M.; Baba, M.; Nishino, H.; Tsuda, H.; Okuyama, T. Cancer Sci. 2004, 95, 448-453.

(21) Raya-Gonzalez, D.; Pamatz-Bolanõs, T.; del Rio-Torres, Rosa E. d.; Martinez-Munõz, R. E.; Ron-Echeverria, O.; Martinez-Pacheco, M. M. Z. Naturforsch., C: J. Biosci. 2008, 63, 922-924.

(22) Yan, X.-T.; Li, W.; Sun, Y.-N.; Yang, S.-Y.; Lee, S.-H.; Chen, J.B.; Jang, H.-D.; Kim, Y.-H. Bioorg. Med. Chem. Lett. 2014, 24, 13971402.

(23) Asada, Y.; Sukemori, A.; Watanabe, T.; Malla, K. J.; Yoshikawa, T.; Li, W.; Kuang, X.; Koike, K.; Chen, C.-H.; Akiyama, T.; Qian, K.; Nakagawa-Goto, K.; Morris-Natschke, S. L.; Lu, Y.; Lee, K.-H. J. Nat. Prod. 2013, 76, 852-857.

(24) Bogorad, L. Annu. Rev. Plant Physiol. 1958, 9, 417-448.

(25) Slade, D.; Ferreira, D.; Marais, J. P. J. Phytochemistry 2005, 66, $2177-2215$

(26) Pouget, C.; Fagnere, C.; Basly, J.-P.; Leveque, H.; Chulia, A.-J. Tetrahedron 2000, 56, 6047-6052.

(27) Mai, H. D.; Nguyen, T. T.; Pham, V. C.; Litaudon, M.; Gueritte, F.; Tran, D. T.; Nguyen, V. H. Planta Med. 2010, 76, 17391742

(28) Soby, S.; Bates, R.; van Etten, H. Phytochemistry 1997, 45, 925929.

(29) Soby, S.; Caldera, S.; Bates, R.; VanEtten, H. Phytochemistry 1996, 41, 759-765.

(30) Goel, A.; Kumar, A.; Raghuvanshi, A. Chem. Rev. 2013, 113, $1614-1640$

(31) Duffy, S.; Avery, V. M. Am. J. Trop. Med. Hyg. 2012, 86, 84-92. (32) Mishra, L. C.; Bhattacharya, A.; Bhasin, V. K. Acta Trop. 2009, 109, 194-198.

(33) Frisch, M. J.; Trucks, G. W.; Schlegel, H. B.; Scuseria, G. E.; Robb, M. A.; Cheeseman, J. R.; Scalmani, G.; Barone, V.; Petersson, G. A.; Nakatsuji, H.; Li, X.; Caricato, M.; Marenich, A. V.; Bloino, J.; Janesko, B. G.; Gomperts, R.; Mennucci, B.; Hratchian, H. P.; Ortiz, J. 
V.; Izmaylov, A. F.; Sonnenberg, J. L.; Williams Ding, F.; Lipparini, F.; Egidi, F.; Goings, J.; Peng, B.; Petrone, A.; Henderson, T.; Ranasinghe, D.; Zakrzewski, V. G.; Gao, J.; Rega, N.; Zheng, G.; Liang, W.; Hada, M.; Ehara, M.; Toyota, K.; Fukuda, R.; Hasegawa, J.; Ishida, M.; Nakajima, T.; Honda, Y.; Kitao, O.; Nakai, H.; Vreven, T.; Throssell, K.; Montgomery, J. A., Jr.; Peralta, J. E.; Ogliaro, F.; Bearpark, M. J.; Heyd, J. J.; Brothers, E. N.; Kudin, K. N.; Staroverov, V. N.; Keith, T. A.; Kobayashi, R.; Normand, J.; Raghavachari, K.; Rendell, A. P.; Burant, J. C.; Iyengar, S. S.; Tomasi, J.; Cossi, M.; Millam, J. M.; Klene, M.; Adamo, C.; Cammi, R.; Ochterski, J. W.; Martin, R. L.; Morokuma, K.; Farkas, O.; Foresman, J. B.; Fox, D. J. Gaussian 16, Revision C.01; Gaussian: Wallingford, CT, 2016.

(34) Autschbach, J. Ab Initio Electronic Circular Dichroism and Optical Rotatory Dispersion: from Organic Molecules to Transition Metal Complexes. In Comprehensive Chiroptical Spectroscopy: Instrumentation, Methodologies, and Theoretical Simulations, Vol. 1; John Wiley \& Sons, 2012; Chapter 21, pp 593-642.

(35) Pupier, M.; Nuzillard, J. M.; Wist, J.; Schlorer, N. E.; Kuhn, S.; Erdelyi, M.; Steinbeck, C.; Williams, A. J.; Butts, C.; Claridge, T. D. W.; Mikhova, B.; Robien, W.; Dashti, H.; Eghbalnia, H. R.; Fares, C.; Adam, C.; Kessler, P.; Moriaud, F.; Elyashberg, M.; Argyropoulos, D.; Perez, M.; Giraudeau, P.; Gil, R. R.; Trevorrow, P.; Jeannerat, D. Magn. Reson. Chem. 2018, 56, 703-715. 